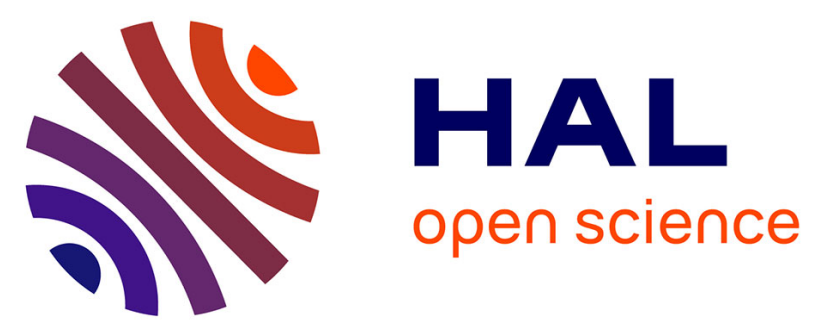

\title{
Error Negativity Does Not Reflect Conflict: A Reappraisal of Conflict Monitoring and Anterior Cingulate Cortex Activity
}

Boris Burle, Clémence Roger, Sonia Allain, Franck Vidal, Thierry Hasbroucq

\section{- To cite this version:}

Boris Burle, Clémence Roger, Sonia Allain, Franck Vidal, Thierry Hasbroucq. Error Negativity Does Not Reflect Conflict: A Reappraisal of Conflict Monitoring and Anterior Cingulate Cortex Activity. Journal of Cognitive Neuroscience, 2008, 20 (9), pp.1637 - 1655. hal-00320988

\section{HAL Id: hal-00320988 https://hal.science/hal-00320988}

Submitted on 28 Oct 2016

HAL is a multi-disciplinary open access archive for the deposit and dissemination of scientific research documents, whether they are published or not. The documents may come from teaching and research institutions in France or abroad, or from public or private research centers.
L'archive ouverte pluridisciplinaire HAL, est destinée au dépôt et à la diffusion de documents scientifiques de niveau recherche, publiés ou non, émanant des établissements d'enseignement et de recherche français ou étrangers, des laboratoires publics ou privés. 


\title{
Error Negativity Does Not Reflect Conflict: A Reappraisal of Conflict Monitoring and Anterior Cingulate Cortex Activity
}

\author{
Borís Burle, Clémence Roger, Sonia Allain, Franck Vidal, \\ and Thierry Hasbroucq
}

\begin{abstract}
Our ability to detect and correct errors is essential for our adaptive behavior. The conflict-loop theory states that the anterior cingulate cortex (ACC) plays a key role in detecting the need to increase control through conflict monitoring. Such monitoring is assumed to manifest itself in an electroencephalographic (EEG) component, the "error negativity" $\left(N_{\mathrm{e}}\right.$ or "error-related negativity" [ERN]). We have directly tested the hypothesis that the ACC monitors conflict through simulation and experimental studies. Both the simulated and EEG traces were sorted, on a trial-by-trial basis, as a function of the degree
\end{abstract}

\section{INTRODUCTION}

In order to adapt to ever-changing environments, animals must continuously alter their behavior. Such flexibility is often assumed to be mediated by "control" mechanisms that adjust information processing to the prevailing context. The way in which control mechanisms are recruited, however, remains obscure. In the last few years, the "conflict-loop theory" (Botvinick, Cohen, \& Carter, 2004; Yeung, Botvinick, \& Cohen, 2004; Botvinick, Braver, Carter, Barch, \& Cohen, 2001; Cohen, Botvinick, \& Carter, 2000; Botvinick, Nystrom, Fissell, Carter, \& Cohen, 1999; Carter, Botvinick, \& Cohen, 1999; Carter et al., 1998) has played an essential role in this field by providing a unified model that aims to account for both neurophysiological and behavioral aspects of control implementation. This model introduced a very simple, though very powerful, concept, namely, "response conflict." Response conflict, measured by the anterior cingulate cortex (ACC), is explicitly defined as the product of the activation of the responses weighted by the inhibitory connections between these responses (Yeung et al., 2004; Botvinick et al., 2001, see also Equation 2). In this type of neural network model, "response activation" refers to the amount of neural activity in the structures involved in response execution. Conflict monitoring has been largely studied in the so-

Aix-Marseille Université, CNRS, Marseille, France of conflict, measured as the temporal overlap between incorrect and correct response activations. The simulations clearly show that conflict increases as temporal overlap between response activation increases, whereas the experimental results demonstrate that the amplitude of the $N_{\mathrm{e}}$ decreases as temporal overlap increases, suggesting that the ACC does not monitor conflict. At a functional level, the results show that the duration of the $N_{\mathrm{e}}$ depends on the time needed to correct (partial) errors, revealing an "on-line" modulation of control on a very short time scale.

called flanker task (Eriksen \& Eriksen, 1974), in which participants must issue a right- or a left-hand response as a function of a target letter (e.g., the letters S or H), flanked by distractors that can be compatible (SSS) or incompatible (HSH) with the target. The implementation of the conflict model for the flanker task has been extensively described by Yeung et al. (2004) and Botvinick et al. (2001). Only the major aspects, and those directly relevant for our purpose, will be described here.

The model is implemented as a three-layer neural network (Cohen, Servan-Schreiber, \& McClelland, 1992): one perceptual layer codes for the target and both distractors, one response layer codes for the two competing responses, and an attentional layer biases processing toward the target. All between-layer connections are excitatory (no between-layer inhibition), whereas all withinlayer connections are inhibitory. To this basic architecture, the conflict modelers added a conflict-monitoring unit that measures on-line the amount of conflict. Generally speaking, the conflict is measured as the energy (Hopfield, 1982 ) in the response layer, defined as:

$$
-\sum \sum a_{i} a_{j} w_{i j}
$$

where $a$ represents the activity of each unit in the layer, indices $i$ and $j$ the different units, and $w_{i j}$ the inhibitory connection between the units $i$ and $j$. 
In the flanker task, where only two possible responses are present, conflict over time, $\mathrm{Co}(t)$, is computed as:

$$
\operatorname{Co}(t)= \begin{cases}-2\left(\operatorname{act}_{\mathrm{H}}(t) \operatorname{act}(t)(-3)\right) & \text { if } \\ 0 & \operatorname{act}_{\mathrm{H}}(t)>0, \operatorname{acts}_{\mathrm{S}}(t)>0 \\ 0 & \text { otherwise }\end{cases}
$$

where $\operatorname{act}_{\mathrm{H}}(t)$-respectively act $(t)$-is the activation level of the unit coding for the $\mathrm{H}$ response-respectively the $\mathrm{S}$ response-at time $t$, and -3 is the (constant) inhibitory connection between the left and the right response units.

Conflict monitoring is assumed to be measurable with functional magnetic resonance imaging (fMRI; Barch, Braver, Sabb, \& Noll, 2000; Botvinick et al., 1999; Carter et al., 1998) and electroencephalography (EEG; West, 2004; Yeung et al., 2004; Rodriguez-Fornells, Kurzbuch, \& Münte, 2002; van Veen \& Carter, 2002; Gehring \& Fencsik, 2001). In the present study, we will concentrate on the EEG correlate: When participants commit an error, one observes a negative component, maximal frontocentrally, called "error negativity" $\left(N_{\mathrm{e}}\right)$ (Falkenstein, Hohnsbein, Hoormann, \& Blanke, 1991), or "errorrelated negativity" (ERN) (Gehring, Goss, Coles, Meyer, \& Donchin, 1993), starting about $30 \mathrm{msec}$ and peaking about 100 msec after the incorrect electromyographic (EMG) activity. Simulations of the model have revealed that conflict is maximal just after the incorrect response activation; its timing is thus comparable to that of the
$N_{\mathrm{e}}{ }^{1}{ }^{1}$ This similarity led Yeung et al. (2004) and Botvinick et al. (2001) to reinterpret the $N_{\mathrm{e}}$ in terms of conflict; conflict that results secondarily in error detection. According to these authors, the time course of the $N_{\mathrm{e}}$ should parallel the time course of the conflict. One way of testing this view is to estimate conflict on a trial-bytrial basis. Because conflict is defined as the degree of coactivation of the responses, it amounts to estimating the degree of overlap between correct and incorrect response activations. One major difficulty is that response activation is a covert process, not directly observable. However, EMG recordings of the muscles involved in responding have proved efficient in revealing such covert activation (e.g., Burle, Allain, Vidal, \& Hasbroucq, 2005; Burle, Possamaï, Vidal, Bonnet, \& Hasbroucq, 2002; Hasbroucq, Possamaï, Bonnet, \& Vidal, 1999; Smid, Mulder, \& Mulder, 1990; Coles, Gratton, Bashore, Eriksen, \& Donchin, 1985): On some trials, although the correct response was given, subthreshold EMG activity in the muscles involved in the incorrect response ("partial error"; see Figure 1A) can be observed. An $N_{\mathrm{e}}$ has been reported to occur just after such partial errors (Burle et al., 2005; Masaki \& Segalowitz, 2004; Vidal, Hasbroucq, Grapperon, \& Bonnet, 2000). Because these partial errors are detectable on a trial-by-trial basis, they offer the unique opportunity to directly evaluate the activation of incorrect responses, not otherwise observable on overt performance. $^{2}$

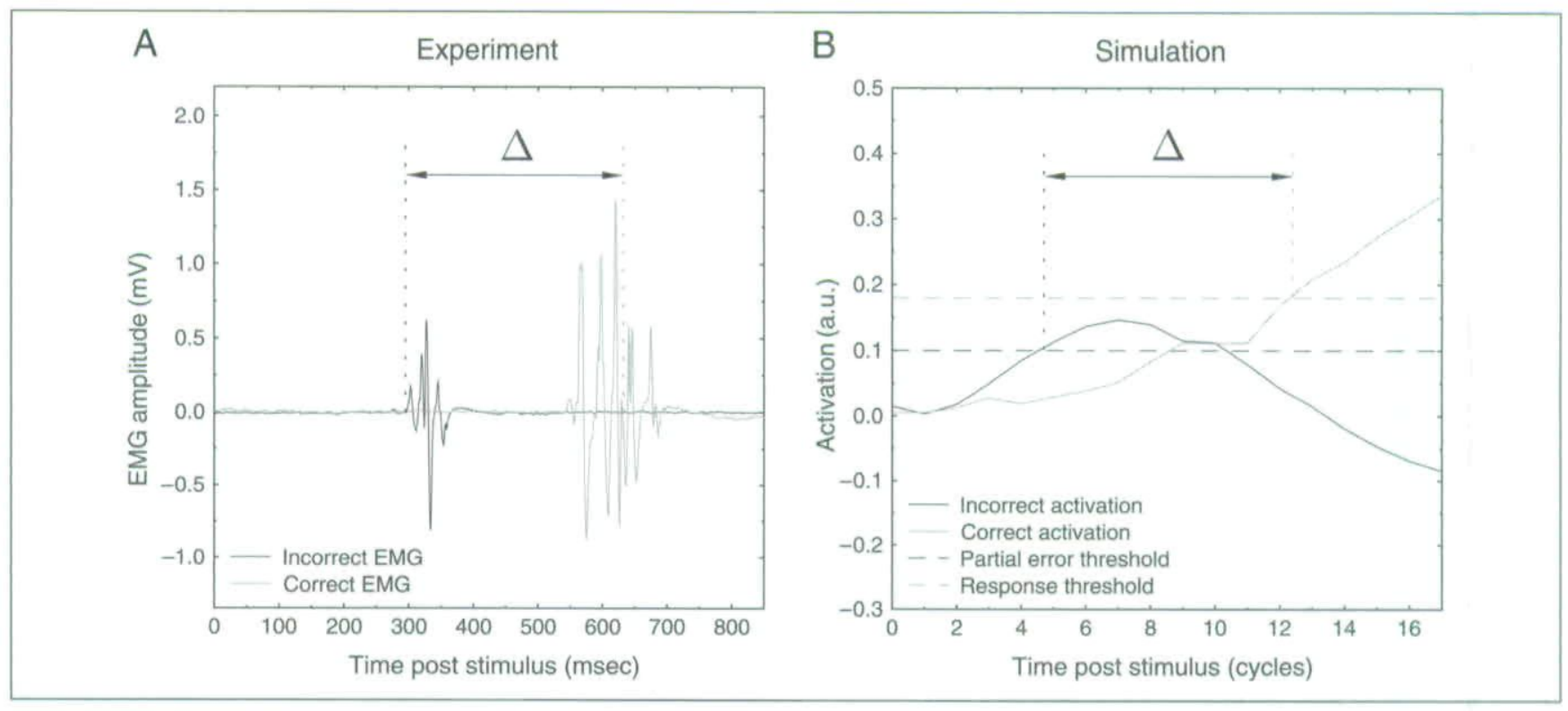

Figure 1. Examples of "partial errors" obtained in the experiment and in the simulation. (A) The left part of the graph presents the EMG activity as a function of time, in the muscles involved in the execution of the incorrect (black) and in the correct responses (gray). The time origin corresponds to stimulus presentation. The vertical black dashed line corresponds to the onset of the partial error, and the vertical gray dashed line to the mechanical response. Although the correct response was given, one can observe an activation of the incorrect response whose amplitude is, however, too low to trigger an overt error. The time interval between the incorrect EMG onset and the correct mechanical response is defined as $\Delta$, and will be used as an index of the temporal overlap between the two response activations. (B) The right part of the figure presents a simulated trial, with the amount of activation (in arbitrary units) of the incorrect (black line) and of the correct (gray line) response as a function of time. The time-zero corresponds to stimulus presentation, the vertical black dashed line corresponds to the onset of the partial error, and the vertical gray dashed line to the timing of the response. The two horizontal long dashed lines correspond to the partial error threshold (black) and to the correct response threshold (gray). 
Importantly, conflict is defined as the degree of coactivation of the two responses (see Equation 2). We thus reasoned that it should depend on the temporal overlap between incorrect and correct response activations that is on the time separating the incorrect from the correct response ( $\Delta$ value on Figure 1$)$. In order to verify the validity of this reasoning, we first ran a simulation study in which trials containing the equivalent of partial errors were analyzed. Because, as the results will show, this assumption was supported, the $\Delta$ value was the main variable of interest. We then compared the simulated data to real, experimental data (see below). In order to compare the simulation results with the experimental ones, we processed the simulation and the EEG data in the same way. To this end, we used the open-source software EEGLAB (Delorme \& Makeig, 2004), which allows single-trial dynamics to be studied (Jung et al., 2001), and hence, reveals more precisely the impact of $\Delta$ value on conflict and $N_{e}$ amplitudes.

\section{METHODS}

\section{Simulation Study}

\section{Simulation Parameters}

The simulation was based on 10 runs (representing 10 participants) of 1000 trials each. All parameters used in the simulation were those used previously in the studies of Yeung et al. (2004) and Botvinick et al. (2001), ${ }^{3}$ except the threshold for "partial errors" (which were not previously considered). In the previous simulations, a response was recorded as soon as one of the two response units reached an activation level of 0.18 . Using this value, a pilot simulation study indicated that a threshold of 0.10 for partial errors gives $10 \%$ to $15 \%$ partial errors, which is equivalent to the percentage usually obtained in empirical studies (Burle et al., 2002; Hasbroucq et al., 1999; Smid et al., 1990; Gratton, Coles, Sirevaag, Eriksen, \& Donchin, 1988). Importantly, changing this value modified the overall number of partial errors, but did not affect the global performance pattern, as the difference in partial errors rate between compatible and incompatible trials remained constant whatever this value (within realistic limits). Thus, the results presented below are not specific to a well-tuned, somewhat arbitrary, value that we have chosen for partial errors threshold.

\section{Data Preprocessing}

Depending on whether the correct or incorrect response unit reached the response threshold first, the trial was classified as correct or erroneous, respectively. Among the correct trials, we checked whether the incorrect response unit reached the partial error threshold before the correct response (it never happened that the incorrect response layer reached the partial error threshold after the correct response). If the incorrect unit reached the predefined threshold of 0.10 , the trial was classified as a partial error trial. In order to have enough trials available for analysis in both the simulation and the experiment, we focused on incompatible trials containing partial errors, as the number of partial errors is higher in incompatible situations (Burle et al., 2002; Smid et al., 1990; Gratton et al., 1988; Coles et al., 1985).

The individual partial error trials were thereafter imported into EEGLAB (Delorme \& Makeig, 2004) for further analysis (see Single-trial Analysis section).

\section{Experimental Study}

\section{Participants}

Ten right-handed participants ( 3 women, 7 men, aged 20 to 31 years, mean age $=25$ years) volunteered for this experiment. They all had normal or corrected-to-normal vision. Before the study, all the participants gave their informed written consent according to the Declaration of Helsinki. They were informed of the purpose and procedure of the experiment before participating.

\section{Experimental Task and Procedure}

The participants performed an Eriksen's flanker task (Eriksen \& Eriksen, 1974) in which they had to respond with a right or a left thumb keypress as fast as possible as a function of a central target letter ( $\mathrm{S}$ or $\mathrm{H}$ ) flanked by two distractors that could be compatible (e.g., SSS) or incompatible (e.g., HSH). The stimuli were presented by a seven-segment light-emitting-diodes display (Lextronic, model SGN-S5, $33 \times 14 \mathrm{~mm}$ ), located $1.5 \mathrm{~m}$ in front of the participant. The stimuli were extinguished with the participants' response. The participants held vertical handgrips on top of which response buttons were fixed.

Participants performed 20 blocks of 128 trials each. After stimulus presentation, they had $1 \mathrm{sec}$ to respond. The next stimulus was delivered $1 \mathrm{sec}$ after the response. All types of trials (HHH, HSH, SHS, and SSS) were equiprobable and presented in a pseudorandom order.

\section{Data Acquisition and Preprocessing}

EEG and EMG activity was recorded with $\mathrm{Ag}-\mathrm{AgCl}$ electrodes (BIOSEMI Active-Two electrodes, Amsterdam). The sampling rate was $1024 \mathrm{~Hz}$ (filters: DC to $268 \mathrm{~Hz}$, $3 \mathrm{~dB} /$ octave). For EEG, we used 64 channels (10-20 system positions). The vertical electrooculogram was recorded by means of two electrodes (same type as EEG) just above and below the left eye, respectively, and the horizontal electrooculogram was recorded with two electrodes positioned over the two outer canthi. EMG was recorded by means of two pairs of electrodes glued to the skin of the thenar eminence above the flexor 
pollicis brevis of each hand. The distance between the two EMG electrodes was $2 \mathrm{~cm}$.

After acquisition, the electrophysiological data were filtered (EEG: high pass $=0.3 \mathrm{~Hz}$, low pass $=100 \mathrm{~Hz}$ and EMG: high pass $=10 \mathrm{~Hz}$ ). Eye movement artifacts were corrected by the statistical method of Gratton, Coles, and Donchin (1983). All other artifacts were rejected after visual inspection of individual traces. The onset of the EMG activity was marked manually after visual inspection. Indeed, although automated algorithms can be useful, visual inspection remains the most accurate technique against which all algorithms are compared (Staude, Flachenecker, Daumer, \& Wolf, 2001; van Boxtel, Geraats, van den Berg-Lessen, \& Brunia, 1993), especially for detecting small changes in EMG activity such as partial errors. Importantly, the experimenters were not aware of the nature of the trial (compatible vs. incompatible) being processed. Furthermore, the EEG signals corresponding to the current EMG were not displayed when detecting EMG onset. Thus, the experimenter was completely blind regarding all the other relevant parameters, and thus, could not, even unwittingly, bias the results.

The trials were classified as correct or erroneous, depending on whether the correct or the incorrect button was pressed first. Among the correct trials, we separated trials containing only one EMG activation on the correct side from trials containing an EMG activation on the incorrect side preceding the correct response (partial error trials; see Figure 1A). Laplacian transformation, as implemented in BrainAnalyser (Brain Products, Munich), was applied to each individual trial to increase the spatial resolution of the EEG (Babiloni, Cincotti, Carducci, Rossini, \& Babiloni, 2001): First, the signal was interpolated with the spherical spline interpolation procedure (Perrin, Pernier, Bertrand, \& Echallier, 1989), hence, the second derivatives in two dimensions of space were computed. We choose 3 for the degree of spline because this value minimizes errors (Perrin, Bertrand, \& Pernier, 1987 ), and the interpolation was computed with a maximum of 15 degrees for the Legendre polynomial. We assumed a radius of $10 \mathrm{~cm}$ for the sphere representing the head, rather than the unrealistic default radius of $1 \mathrm{~m}$ assumed by BrainAnalyser. With such a realistic radius, the most suitable unit is $\mu \mathrm{V} / \mathrm{cm}^{2}$. The individual Laplacian transformed trials were imported in EEGLAB for further analysis.

\section{Temporal Overlap Estimation}

In order to compare temporal overlap in simulated and experimental data, we choose a functionally equivalent measure for both. This measure, the $\Delta$ value depicted on Figure 1, corresponds to the time between the onset of the partial error and the moment of the correct response (Figure 1A) for the experimental data, and to the "time" (represented in "cycles") between the par- tial error threshold $(0.10)$ and the correct response one (0.18; Figure 1B) for the simulations. In both cases, a greater $\Delta$ value indicates a longer time between the incorrect response activation and the correct response, revealing a lower temporal overlap. In the following, we will use the $\Delta$ value as a measure of temporal overlap.

\section{Single-trial Analysis}

EEG signal analysis normally relies on averaging techniques. Averaging, however, induces a considerable loss in the dynamics of the process of interest (Jung et al., 2001), as will also be exemplified below. We therefore resorted to the event-related potential image ("ERP image") technique, implemented in the EEGLAB software (Delorme \& Makeig, 2004), allowing one to visualize brain activity without averaging. This technique has been detailed elsewhere (Jung et al., 2001), and will only be briefly described here. Note that this technique was applied not only to the Laplacian-transformed EEG data but also to the simulated data. To construct the individual ERP images, the trials are first sorted based on a relevant measure. In our case, for both the simulation and the experimental data, the time-zero corresponds to the onset of the partial error, and the trials are arranged by increasing $\Delta$ values. The trials are then plotted as parallel colored lines. The result is a "raster-like" plot, with the $x$-axis representing time, the $y$-axis representing the arranged trials, and a color code indicating the intensity of the signal for each trial and each time point. On all figures, the vertical black line indicates the onset of the partial error, and the S-shaped one indicates the correct response. Below each raster-like plot, the average of the traces is represented as a function of time, hence, giving an estimate of the activity under analysis. For the simulation, the blue color indicates conflict (positive values), whereas for the experimental data, blue represents negative polarity. This was done to improve the comparability between the conflict and the $N_{\mathrm{e}}$, both appearing in blue in the ERP images.

ERP images have been computed for each participants: For the experimental data, the ERP images are based on the individual Laplacian-transformed trials. For the simulation, this was performed on the individual activation function and/or the computed conflict traces. Besides individual subject representations, we sought for a population-based representation of the data. One way of doing so is to put all the trials of all the subjects in the same ERP image. This approach has some strengths and limitations. The main interest is that one can visualize all trials of all subjects. However, the within-subject variance, in amplitude and in $\Delta$ values, is confounded with the between-subject variance: For example, subjects with short $\Delta$ values will mainly contribute to the lower part of the ERP image, whereas subjects with long $\Delta$ values will mainly be present on the upper part. A similar bias holds for amplitude. Finally, data represented 
in this way are not comparable to more traditional grandaveraged representation. One alternative approach is to built a "grand-averaged" ERP image by averaging the individual ERP images (see Appendix A for further details). In this case, all subjects have the same weight at every points of the ERP image, allowing one to visualize the impact of $\Delta$ values on the population without bias induced by between-subject variability. One disadvantage, however, is that we do not see, in this case, "real" individual trials, but averaged ones for a given normalized $\Delta$ value. Because these two methods have complementary strengths and shortcomings, we present the two representations along with individual ERP images. Because these various approaches led to very consistent results, despite their different strengths and drawbacks, we considered that the features expressed on these analyses could not be artifacts induced by any of the methods.

As recommended by Jung et al. (2001), the ERP images have been smoothed, with a smoothing width set at about $10 \%$ of the number of trials. One exception, however, is to be noted for the primary motor cortices activations (Figure 7C and D): Because the activities of interest were of small amplitude leading to a lower signalto-noise ratio, the smoothing step was set at about $20 \%$.

\section{Statistical Analysis}

ERP images, although very informative, do not allow one to statistically validate the observed features. To do so, both the simulated and experimental trials were binned into different classes depending on the $\Delta$ interval. The classes were of equal width.

For the simulation, four classes of three cycles were retained that contained the largest number of trials for a reliable estimate of the conflict amplitude: Class $1=$ from 2 to 4 cycles, Class $2=$ from 5 to 7 cycles, Class $3=$ from 8 to 10 cycles, and Class $4=$ from 11 to 13 cycles. The conflict signals obtained on each trial were then averaged, time-locked to the partial error onset for each class, and each run separately. The peak of conflict was determined as the maximum value in a window starting from the partial error onset and lasting 20 simulation cycles. We measured the peak and latency of this peak. Besides these "static" parameters, we investigated the dynamic aspects of conflict by studying how conflict develops. To do so, we analyzed the rising slope of the conflict by fitting a linear regression to the conflict signal in a window from the partial error onset to the fourth cycle following the partial error onset.

For the EEG data, the same method was applied: Individual trials were binned as a function of the time separating the incorrect EMG onset from the correct response. Four classes that contained the larger number of trials (Class 1: from 101 to $150 \mathrm{msec}$, Class 2: from 151 to $200 \mathrm{msec}$, Class 3: from 201 to 250 , and Class 4: from 251 to $300 \mathrm{msec}$ ) were retained. The EEG activity was averaged for each participant and for each class sepa- rately. As for the simulation, the parameter used to estimate the $N_{\mathrm{e}}$ was the amplitude of the peak of the $N_{\mathrm{e}}$ (defined as the difference between the positive peak occurring just after the partial error-between 10 and $50 \mathrm{msec}$ - and the following negative peak-between 50 and $150 \mathrm{msec}$ ). We also analyzed how the $N_{\mathrm{e}}$ develops in time by fitting a linear regression on the rising slope in a time window from 50 to $100 \mathrm{msec}$.

The statistical analysis involved either Student's $t$ tests, for comparisons between two means, or analyses of variance (ANOVAs), for comparisons of more than two means. When ANOVAs were performed, the error term was always the interaction between the factor "participants" and the factor under analysis. Percentages and rates cannot be tested directly with parametric methods because their mean and variances are closely related. However, the arcsine transform $\left(p^{\prime}=\operatorname{asin} \sqrt{p}\right.$, with $p$ being the rate under analysis) has proved to be efficient in stabilizing the variances (Winer, 1971), and was therefore used consistently for each analysis involving rates.

\section{RESULTS}

\section{Behavioral Data}

The overall percentage of errors was $5.2 \%$. The number of overt errors was higher in the incompatible condition $(7.5 \%)$ than in the compatible one (2.9\%) $[t(9)=6.55$, $p<.001]$. For correct trials, reaction time (RT) was longer when the flankers were incompatible ( $416 \mathrm{msec})$ than when they were compatible $(386 \mathrm{msec})[t(9)=$ $15.36, p<.001]$.

The number of partial error trials (see Figure 1) was higher in the incompatible situation (21.7\%) than in the compatible one (14.3\%) $[t(9)=5.78, p<.001$, in line with previously reported empirical data; Burle et al., 2002; Smid et al., 1990; Coles et al., 1985) (more behavioral data, and a deeper comparison between the experimental and the simulated results on these issues, are presented in Appendix B).

\section{Comparison between Conflict and $N_{\mathrm{e}}$ Timing as a Function of $\Delta$}

In previous simulation studies (Yeung et al., 2004; Botvinick et al., 2001), conflict was analyzed time-locked to the stimulus and/or to the response. Since here we introduce partial errors to the simulation, we first verified that a peak of conflict was indeed obtained just after the partial error. Figure $2 \mathrm{~A}$ presents the grand-averaged $N_{\mathrm{e}}$ time-locked to partial error onset. As already reported (Burle et al., 2005; Allain, Carbonnell, Burle, Hasbroucq, \& Vidal, 2004; Masaki \& Segalowitz, 2004; Vidal et al., 2000), a clear $N_{\mathrm{e}}$ was apparent just after partial errors. Furthermore, the $N_{\mathrm{e}}$ observed after partial errors is clearly localized fronto-centrally (see Figure 8). Figure 2B shows the grand-averaged conflict obtained in 
A

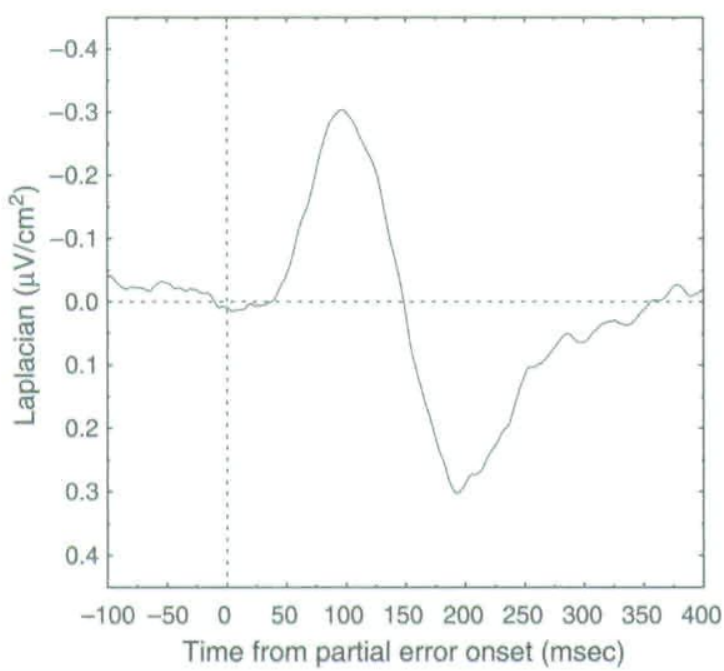

B

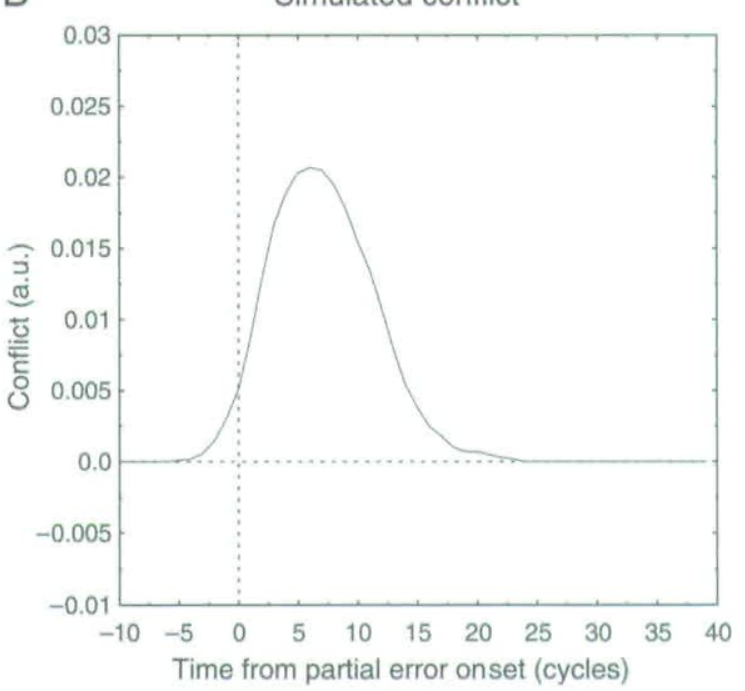

Figure 2. Comparison between grand-averaged $N_{e}$ and conflict. (A) Amplitude of the Laplacian transformed $N_{e}$ induced by partial errors as a function of time. The time-zero corresponds to the partial error onset. A clear $N_{\mathrm{e}}$ can be observed starting about 30 msec after incorrect EMG onset and peaking about $100 \mathrm{msec}$ after it. Furthermore, its topography is well localized fronto-centrally (see Figure 8 for the topography of this wave). (B) Grand-averaged conflict as a function of time. Time-zero corresponds to crossing of the partial error threshold. The conflict is clearly maximal just after the partial error, and its timing nicely fits that of the $N_{\mathrm{e}}$. This confirms that the $N_{\mathrm{e}}$ on partial errors is a valid measure for testing the conflict model predictions.

the simulations when time-locked to the partial error. As anticipated, the conflict is maximal just after the partial error, validating the comparison between $N_{\mathrm{e}}$ and conflict on partial error trials.

Figure 3 show the single-trial dynamics of the $N_{\mathrm{e}}$ and of conflict. Panels A and $\mathrm{C}$ present the grand-averaged ERP images, whereas Panels B and D present all trials of all subjects plotted together. Panels E, F, and G present single-subject ERP images (see above for a presentation of these different approaches). Time-zero corresponds to the partial error onset in all cases, and the S-shaped black line indicates the occurrence of the correct response. One striking feature that appears in the comparison of the two graphs is the difference in timing of the conflict and the $N_{\mathrm{e}}$ : Although the simulated conflict shows an S-shape very similar to the correct response one, the $N_{\mathrm{e}}$ seems better time-locked to the partial error onset. It seems, however, that the width of the $N_{\mathrm{e}}$ increases as $\Delta$ increases. To clarify these points, we binned the trials into different classes varying in term of $\Delta$ (see the Methods section).

The grand-average EEG data for each class is presented on Figure 4A. An ANOVA conducted on the peak amplitude revealed an effect of $\Delta[F(3,27)=3.91$, $p<.05]$ : The smaller the $\Delta$, the smaller the $N_{\mathrm{e}}$. The latency of the peak was also affected by $\Delta[F(3,27)=$
7.42, $p<.001]$, with the peak occurring earlier for small $\Delta$ than for large $\Delta$. In contrast, no effect of $\Delta$ was observed on the rising slope of the $N_{\mathrm{e}}[F(3,27)=1.34$, $p=.28$ ]. Therefore, the latency effect is simply a consequence of the fact that, with the rising slope being the same but the amplitude higher, the peak is reached later.

The same analysis was performed on conflict (Figure $4 \mathrm{~B})$. ANOVAs revealed a clear effect of $\Delta$ on the amplitude of conflict $[F(3,27)=400.24, p<.001]$ : The smaller the $\Delta$, the higher the peak of conflict. The latency of the peak of conflict was also affected by $\Delta[F(3$, $27)=409.17, p<.001]$, with a peak of conflict occurring later as $\Delta$ increased. Finally, the rising slope of the conflict was also steeper when $\Delta$ was low than when it was high $[F(3,27)=20.72, p<.001]$. It therefore appears that the amplitude of conflict decreases as $\Delta$ increases. The dynamics of the conflict are also affected because the rising slope is sensitive to $\Delta$.

\section{Alternative Measure of Conflict}

Although the sensitivity of the $N_{\mathrm{e}}$ to $\Delta$ is opposite to that of conflict as defined in the model, conflict seems to last longer when $\Delta$ increases. We therefore evaluated whether a slight modification of the conflict computation could account for the data: Instead of assuming that

Figure 3. ERP images of the $N_{\mathrm{e}}$ and of the simulated conflict. The trials containing a partial error were sorted as a function of increasing $\Delta$ values. The vertical black line indicates the onset of the partial error and the S-shaped black line indicates the moment of the correct response. (A and B) Grand-average ERP images of $N_{e}$ and simulated conflict. (C and D) Grand-average ERP image containing all trials of all "subjects' for the $N_{e}$ and the simulated conflict, respectively. (E, F, and G) Examples of individual ERP images. Whatever the representation, the conflict appears more time-locked to the correct response, contrary to the $N_{e}$, which appears more time-locked to the incorrect response activation. 


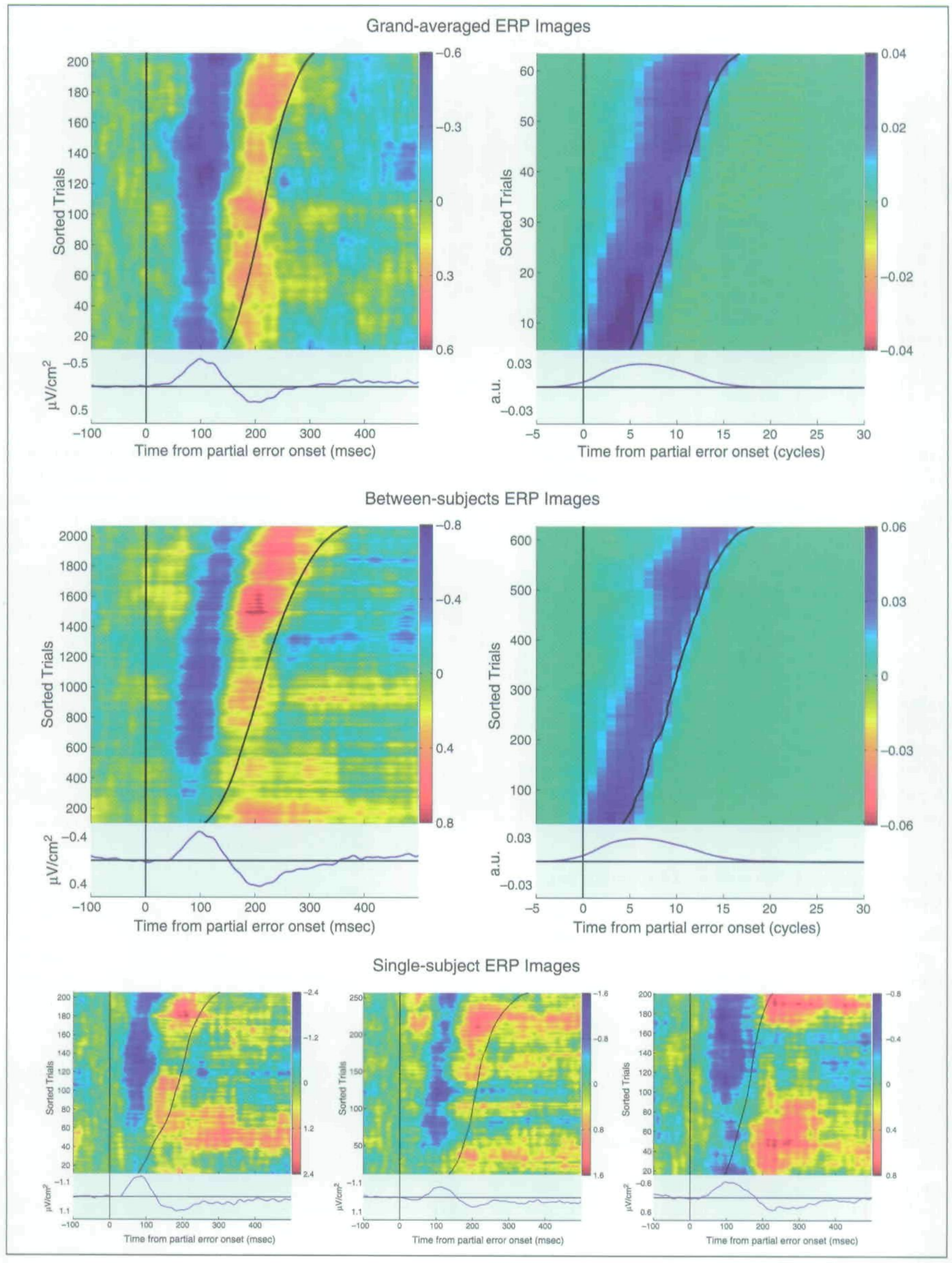



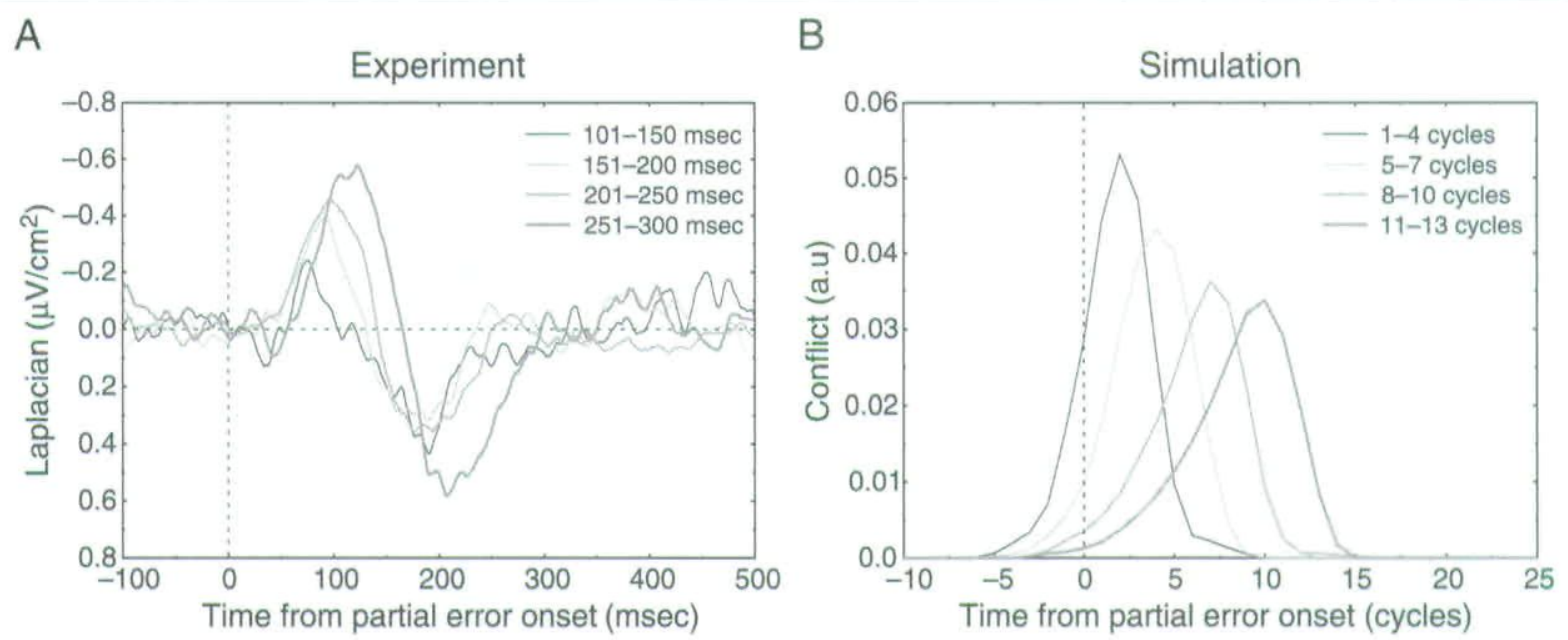

Figure 4. (A) Classes analysis: Amplitude of the $N_{\mathrm{e}}(30 \mathrm{~Hz}$ low-pass filtered) as a function of time, for the four classes. Time-zero corresponds to the onset of the partial error (see Figure 1). The amplitude of the $N_{e}$ increases as $\Delta$ increases. (B) Amount of conflict as a function of time for the four classes. Time-zero corresponds to the moment at which the partial error threshold $(0.10)$ was crossed. The amplitude of the conflict, measured at the peak, decreases as $\Delta$ increases, therefore invalidating the proposition that the $N_{\mathrm{e}}$ reflects conflict.

conflict at time $t$ is the degree of coactivation of the two responses at time $t$, let us consider that it reflects the integration of such coactivation over time, that is, conflict at time $t$ could be:

$$
\operatorname{Co}(t)=\sum_{0}^{t}-2\left(\operatorname{act}_{\mathrm{H}}(t) \operatorname{act}_{\mathrm{S}}(t)(-3)\right)
$$

As a matter of fact, this quantity is assumed to be used for sequential adjustments (Botvinick et al., 2001; see, however, Burle et al., 2005). We therefore estimated the surface under the conflict curve for each simulated participant and for each class. This analysis revealed no change in conflict as a function of class $(F<1)$. Therefore, even the integrated conflict does not increase as $\Delta$ decreases, contrary to the $N_{\mathrm{e}}$.

\section{Conflict and the Dynamics of Incorrect and Correct Response Activation}

The above presented results are in clear disagreement with the interpretation of the $N_{\mathrm{e}}$ in terms of conflict. More specifically, the amplitude of the $N_{\mathrm{e}}$ does not parallel the degree of conflict. To better understand where the discrepancy comes from, we studied in more detail the activation dynamics of the incorrect and correct responses.

\section{Conflict and the Amount of Incorrect Response Activation}

When referring to Equation 2, it appears that conflict does not only depend on the temporal overlap between the two responses but also on the amount of incorrect response activation (see simulation 5 of Yeung et al., 2004). Differences in incorrect response activations might explain the observed discrepancy. To evaluate this, we averaged the simulated activation function of the incorrect responses, time-locked to the partial error onset for the four classes retained above. We processed the experimental data in a similar way: We took the size of the partial error EMG burst as an index of the incorrect response activation. To do so, the EMG bursts were rectified and then averaged separately for the same four classes used for the $N_{\mathrm{e}}$. Figure 5 presents the results of this analysis. As one can see, for both the experimental (Figure 5A) and simulated (Figure 5B) data, the amount of incorrect response activation was greater when $\Delta$ was high. Indeed, the size of the partial error (i.e., the amount of EMG) increased as the interval between the incorrect and correct response activation got larger $[F(3,27)=24.43, p<.001]$. The linear component was also significant $[F(1,9)=46.67, p<.001]$. The simulation results were similar: The amount of incorrect response activation increases as $\Delta$ increases $[F(3$, $27)=71.45, p<.001$; linear component: $F(1,9)=$ $160.1, p<.001]$. These results have two important consequences. The first one is that, as anticipated, conflict is more sensitive to temporal overlap than to the mere amount of incorrect response activation. Indeed, the maximum conflict was obtained in the situation where the activation of the incorrect response was the lowest. Second, because the same increase in incorrect response activation is observed in the experimental and simulated data, the difference between the $N_{\mathrm{e}}$ amplitude and conflict cannot be explained by a difference in response activation.

\section{Dynamics of Correct and Incorrect Responses Activations}

We further explored the discrepancy between conflict and the $N_{\mathrm{e}}$, by investigating the "empirical conflict" present in the experimental data, that is, the degree of 


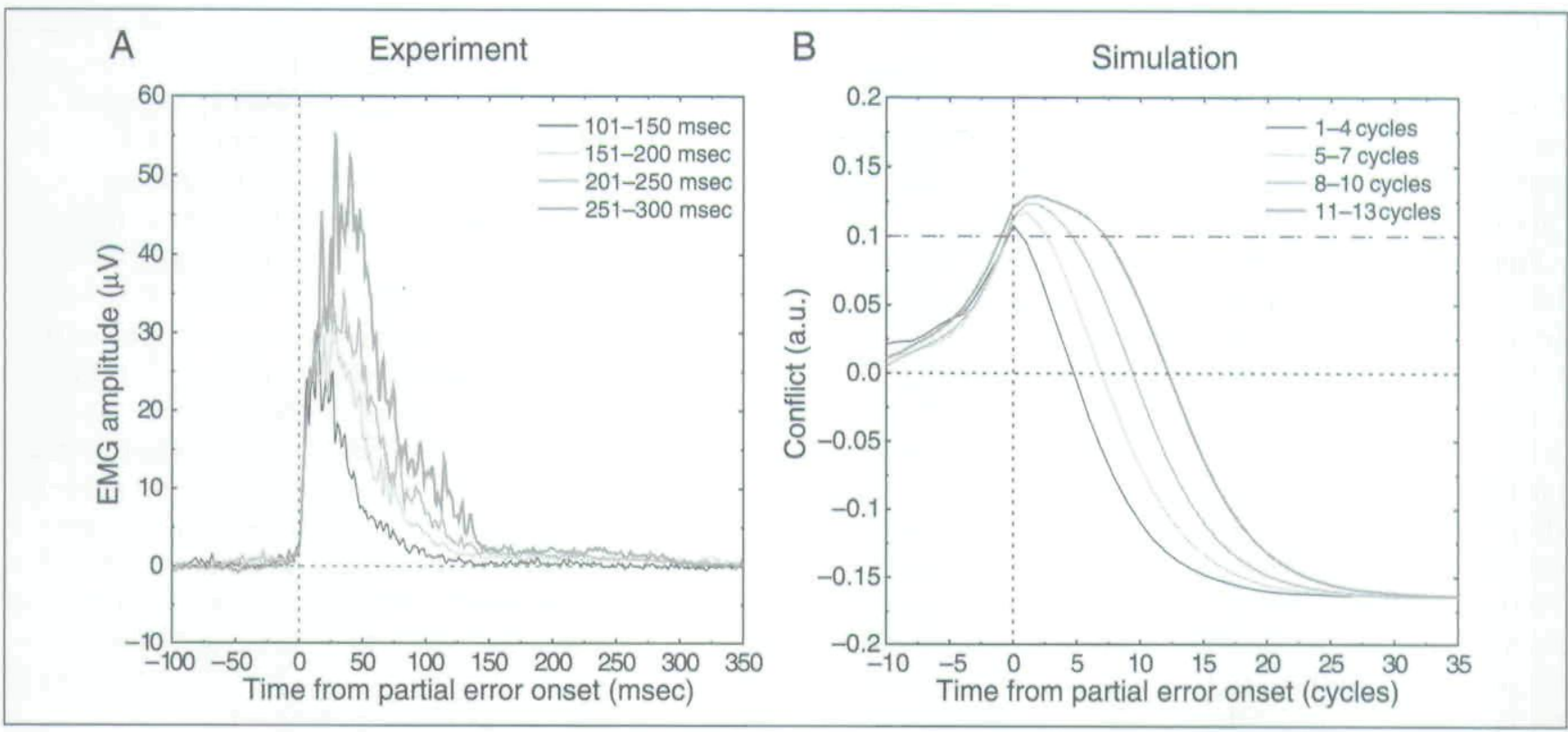

Figure 5. Amount of incorrect response activation as a function of $\Delta$. (A) Grand-averaged full-wave rectified EMG for the four classes retained. The amount of EMG increases as $\Delta$ increases. (B) Grand average of incorrect response activation in the simulation for the four classes. As for EMG, the amount of incorrect response activation increases as $\Delta$ increases. The horizontal line at 0.10 indicates the threshold for a partial error.

coactivation of the alternative responses. We first analyzed the dynamics of response activation as estimated at the EMG level. Indeed, EMG seems a consensual measure of response activation because, according to Yeung et al. (2004, p. 937), "Gehring and Fencsik (1999) have reported $[. .$.$] that the ERN coincides with periods$ of coactivation of the correct and incorrect responses as measured through electromyography (EMG)." A similar argument was also put forward by Botvinick et al. (2001, p. 635). To evaluate the "empirical conflict" we applied Equation 2 to the averaged EMG traces, time-locked to the incorrect EMG onset (Figure 6A).

The two averaged EMG activities overlap around $100 \mathrm{msec}$ after partial error onset, which replicates previous results (Masaki \& Segalowitz, 2004; Gehring \& Fencsik, 1999). Figure 6B presents the estimated conflict, after applying Equation 2 to these averaged EMG data. The estimated "conflict" starts just after the
A

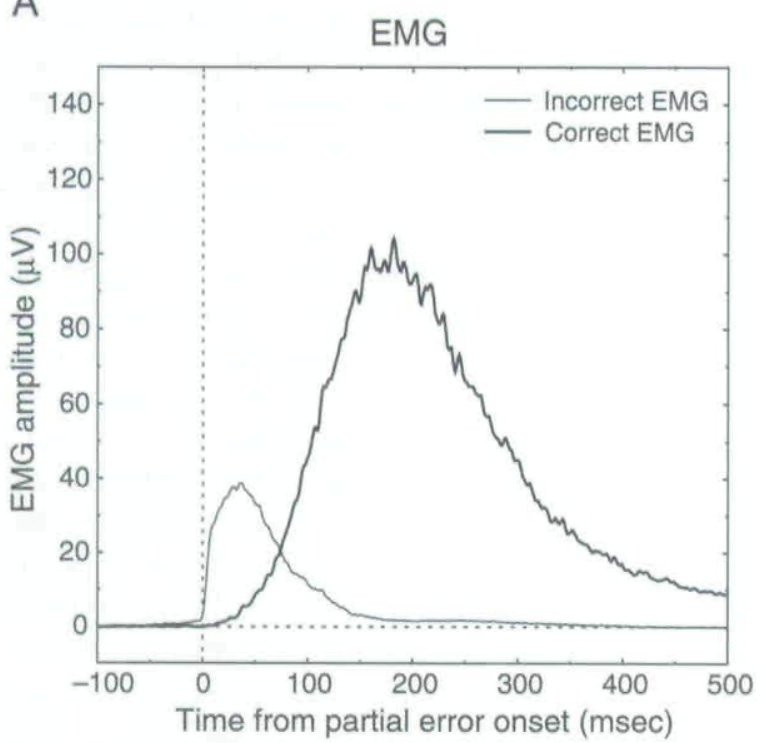

B

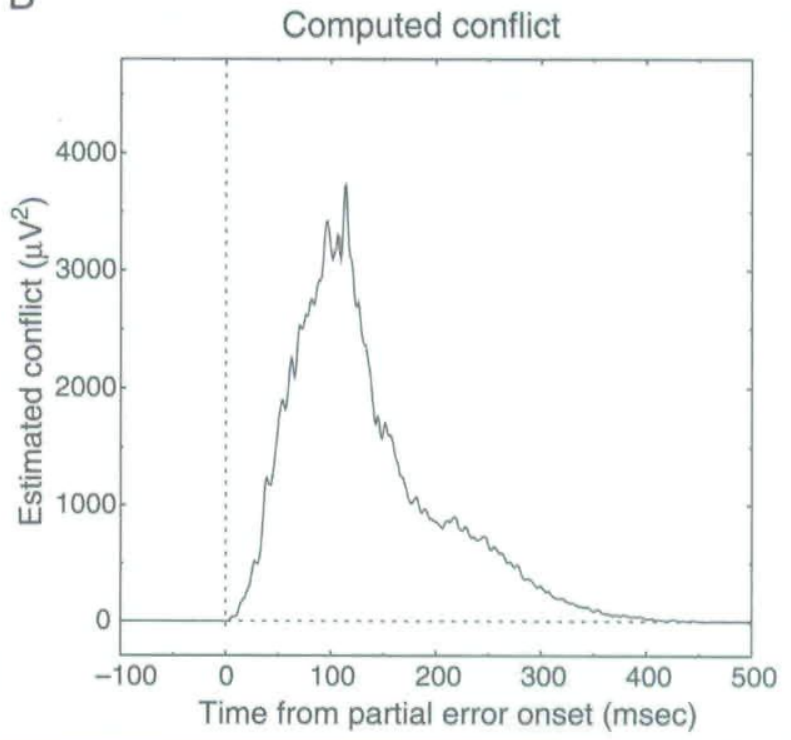

Figure 6. Empirical coactivation and conflict measured on the averaged traces. (A) Temporal overlap of incorrect and correct response activation as measured with averaged EMG. The two responses appear to be coactivated around $100 \mathrm{msec}$ after partial error onset. (B) Empirical conflict obtained by applying Equation 2 to the averaged EMG activities. The empirical conflict nicely fits the prediction of the model, with conflict occurring shortly after the incorrect response activation, and peaking around $100 \mathrm{msec}$. This similarity, however, is an artifact due to averaging. 
partial error onset and peaks about $100 \mathrm{msec}$ after it. This timing almost perfectly fits the one of the $N_{\mathrm{e}}$ reported on Figure 2A. This replicates the data of Gehring and Fencsik (1999). This apparent similarity is, however, an averaging artifact (Callaway, Halliday, Naylor, \& Thouvenin, 1984), as demonstrated below.

The first row of Figure 7 presents the ERP images for the incorrect (Panel A) and correct (Panel B) EMG. A striking effect emerges from these ERP images: Although the duration of the EMG burst is longer when the interval between the incorrect and the correct response increases (see Figure 5), the degree of overlap between the two EMG activities is virtually null, except maybe when the interval is very small. Thus, as long as response activation is evaluated at the EMG level (Yeung et al., 2004), the incorrect and correct response activations did not overlap in any of the trials, and the conflict is null in this dataset. The apparent overlap observed on Figure 6 stems from the fact that the slowest incorrect activations occur later than the fastest correct ones, but not on the same trials. This exemplifies the hazards of averaging procedures because, although we do observe "conflict" on averaged data that very nicely fit the predictions of the model, there is no "conflict" (as defined in the model...) at all, on any of the trials, in this dataset.

One may argue, however, that even if coactivation does not occur at the EMG level, it might well be present at the central level. We thus analyzed the activity of the primary motor cortices contralateral to the incorrect and correct responses. Indeed, thanks to Laplacian estimation, it recently became possible to estimate the activation of the two primary motor cortices separately in choice RT tasks (Praamstra \& Seiss, 2005; Tandonnet, Burle, Vidal, \& Hasbroucq, 2003; Vidal, Grapperon, Bonnet, \& Hasbroucq, 2003; Taniguchi, Burle, Vidal, \& Bonnet, 2001; see Burle, Vidal, Tandonnet, \& Hasbroucq, 2004 for an overview).

The second row of Figure 7 presents the cortical activation of the incorrect response, that is, the activity of the primary motor cortices contralateral to the partial error (left column) and the cortical activation of the correct one, that is, the activity of the primary motor cortices contralateral to the correct response. The topographies of these activities, along with that of the $N_{\mathrm{e}}$, are presented in Figure 8.

Because the signal-to-noise ratio of EEG is much lower than that of EMG, the results have to be interpreted with some caution. Some systematic patterns emerge nonetheless: The incorrect response activation, as measured at the primary motor cortices contralateral to the incorrect response, starts just before incorrect EMG onset (blue strip on Figure 7C), peaks shortly after EMG onset, and then decreases back to baseline, or slightly below. It seems that the incorrect response activation is over about 30-40 msec after incorrect EMG onset. The correct response activation (Figure 7D) follows the $\Delta$ value and starts later as this value increases. More im- portantly, the activation of the correct response clearly starts after the end of the incorrect response activation, and there seems to be virtually no overlap between the incorrect and correct response activation. Thus, here again, no conflict, as defined in the model, seems to emerge at the primary motor cortices level. We shall return to this absence of "conflict" in the Discussion.

Another interesting aspect is to be noted: When comparing the dynamics of the incorrect response activation and of the $N_{\mathrm{e}}$ (see Figure 3A), it appears that the $N_{\mathrm{e}}$ invariably starts around the end of the incorrect response activation. Because it starts after the end of the incorrect activation, the $N_{\mathrm{e}}$ cannot be responsible for the interruption of the incorrect response activation, as this incorrect response activation had necessarily been detected earlier in order to be stopped. Thus, the $N_{\mathrm{e}}$ cannot reflect the detection of this incorrect response activation, invalidating a simple error-detection mechanism (see Gehring \& Fencsik, 1999 for similar arguments on EMG).

For the sake of comparison, we also plotted the ERP images of the incorrect and correct response simulated activations. The third row of Figure 7 presents the singletrial dynamics of the incorrect (Panel E) and correct response (Panel F) activations obtained in the simulation. The incorrect response starts being activated just before the partial error onset and lasts almost until the correct response. The incorrect response starts being deactivated when the correct response starts being activated. The conflict, depicted on Figure 3B, thus peaks just before the correct response, when the two responses are still activated and are maximally competing.

\section{DISCUSSION}

In the last few years, the conflict-loop theory (Botvinick et al., 1999, 2001, 2004; Yeung et al., 2004; Cohen et al., 2000; Carter et al., 1998, 1999) has played a key role in cognitive control research since it proposed a unified model that aimed to account for both behavioral and neurophysiological data. The model introduced a very simple, although elegant, concept: Response conflict is defined as the degree of coactivation of the possible responses. It has been argued that monitoring conflict allows, without any "clever" cognitive system, to detect failures in ongoing processing, and to adjust subsequent behavior. The model explicitly relates conflict monitoring with ACC activity as observed with metabolic measures, and through specific EEG components. The main EEG component related to conflict is the $N_{\mathrm{e}}$. We will first discuss the relationship between the $N_{\mathrm{e}}$ and conflict, and then discuss the very concept of conflict as a relevant notion for cognitive control.

\section{$N_{\mathrm{e}}$ and Conflict}

The $N_{\mathrm{e}}$ has played an essential role in the development of the conflict model (Botvinick et al., 2001, 2004; Yeung 


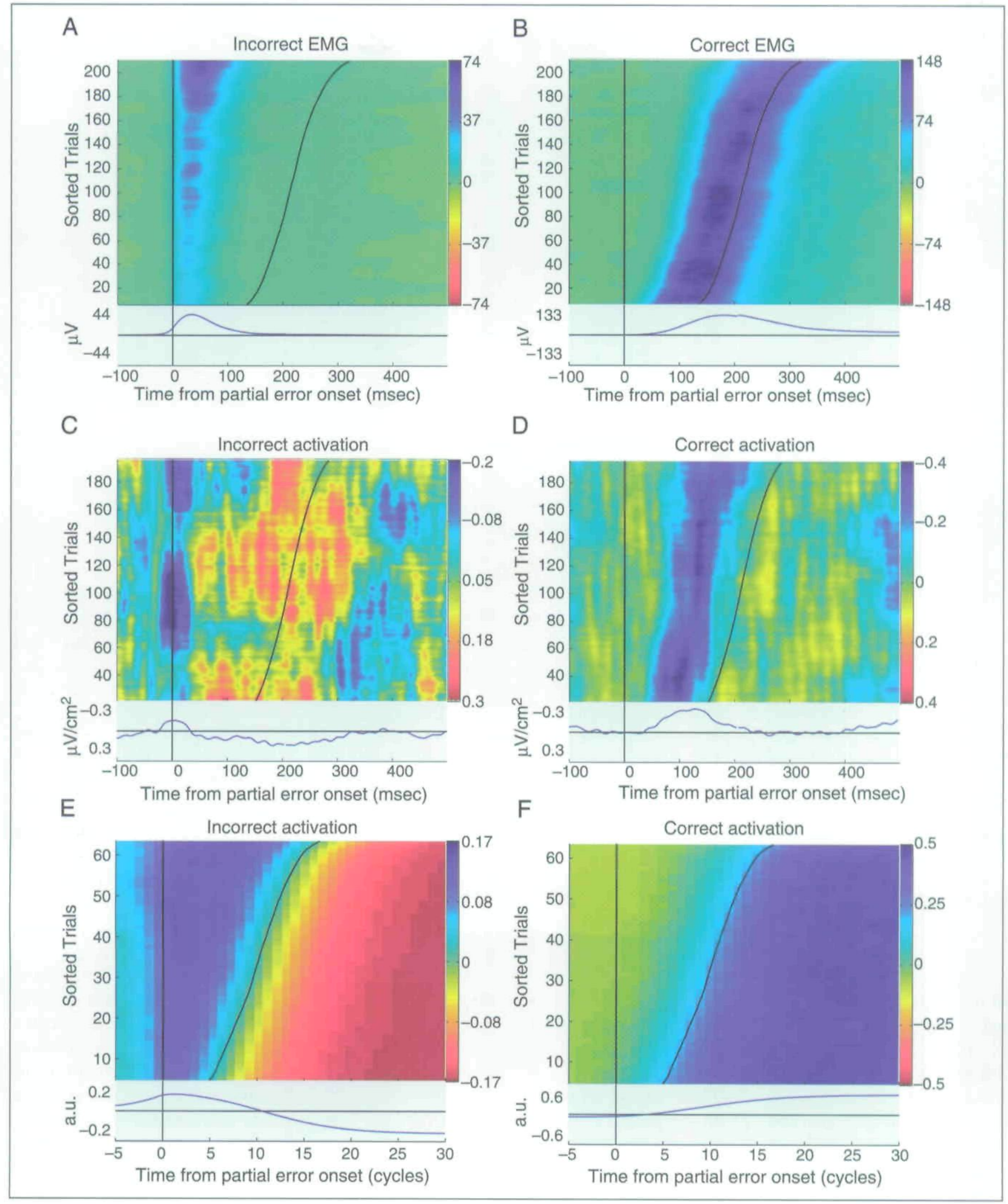

Figure 7. Single-trial analysis of response activation. Incorrect (left column) and correct (right column) response activations as a function of $\Delta$, for the experiment as estimated by EMG amplitude (first row), or at the cortical level through the Laplacian estimated over the primary motor cortices (second row), and for the simulation (third row). 


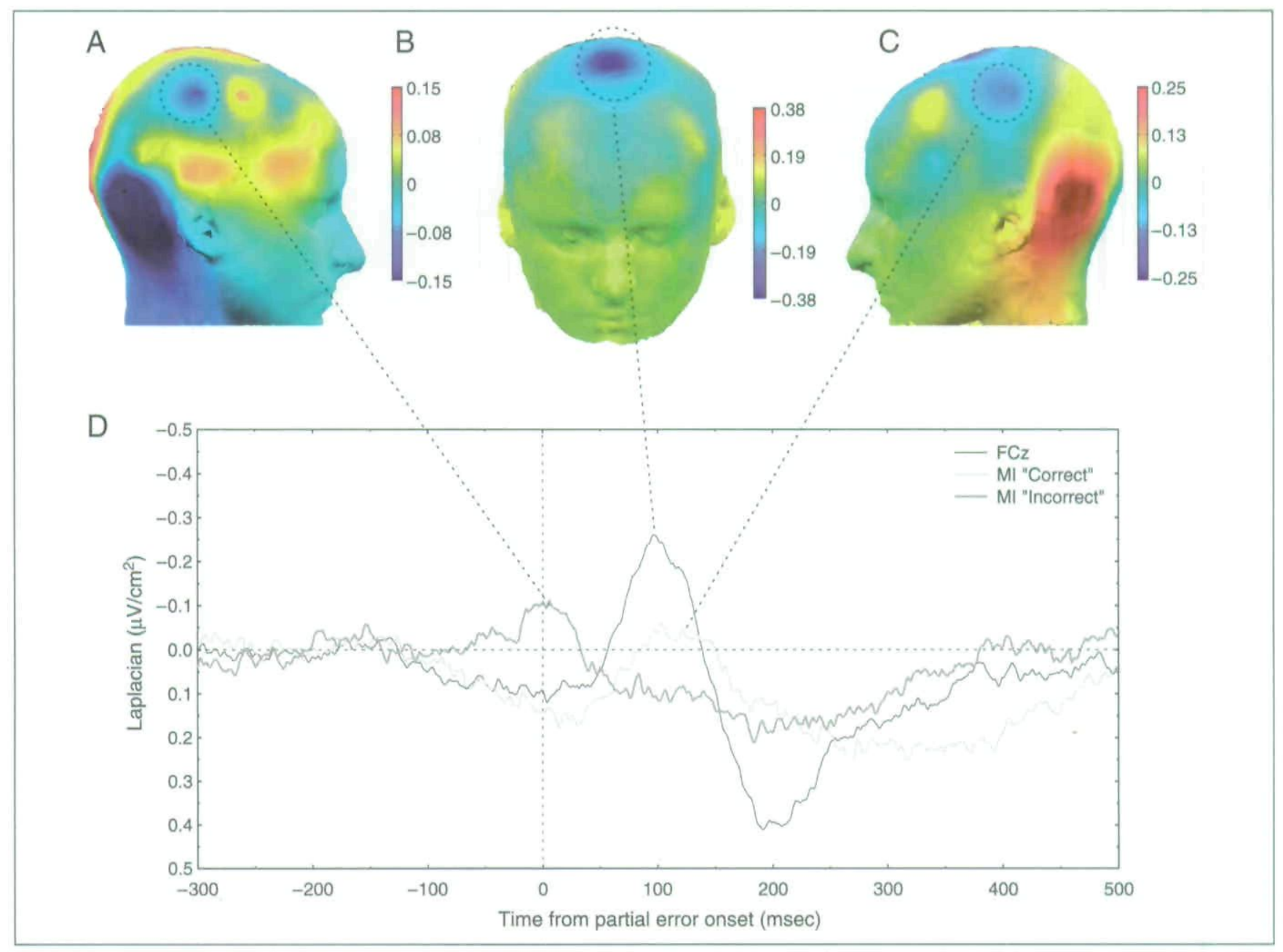

Figure 8. Topography and time course of the activity of interest. For sake of simplicity, activity related to the two responses were averaged after mirroring activity for the left response: Activity recorded over right electrodes for the left responses were projected to their corresponding left electrodes, and vice-versa. Thus, C3 corresponds to the primary motor cortices contralateral to the correct response, whereas C4 corresponds to the primary motor cortices contralateral to the incorrect response (i.e., the partial error). Except when noted, the baseline for the maps have been taken between -500 and $-100 \mathrm{msec}$. (A) Laplacian (CSD) map obtained at incorrect activation time ( 9 msec after EMG onset). One observes a clear activity localized over C4, that is at the level of the primary motor cortex involved in the incorrect response. (B) Laplacian map obtained at the peak of the $N_{\mathrm{e}}$. The topography is clearly fronto-central, compatible with source(s) in the ACC and/or in the SMA. (C) Laplacian map at the time of the correct response activation. One can observe a clear activity nicely localized over $\mathrm{C} 3$, that is, over the primary motor cortices contralateral to the correct response. Note that, because of the positivity starting just before partial error onset (likely reflecting an inhibition; see Burle et al, 2004), the baseline was taken between -50 and 50 msec for the map. (D) Time courses of the above-described activities.

et al., 2004). Reciprocally, the conflict model has been extremely influential in the $N_{\mathrm{e}}$ literature because a lot of studies have used the conflict model as a general framework for interpreting their results. However, although several studies have tested the conflict interpretation of ACC activity observed in metabolic research (Brown \& Braver, 2005; Egner \& Hirsch, 2005), few studies have explicitly addressed the conflict model hypothesis on the $N_{\mathrm{e}}$, apart from the conflict modelers themselves (Yeung et al., 2004; Botvinick et al., 2001; see Carbonnell \& Falkenstein, 2006 for an exception). Here, we directly tested the conflict interpretation of the $N_{\mathrm{e}}$ by estimating the amount of conflict on a trial-by-trial basis by analyzing partial error trials (Figure 1) that allow, on a single-trial basis, to track the activation of incorrect and correct responses. According to the definition of con- flict, we reasoned that conflict should depend on the temporal overlap between the two response activations that we measured through the $\Delta$ value. A simulation confirmed that the amount of conflict depends on the $\Delta$ between the two response activations: The smaller the $\Delta$ between the incorrect and correct response activations, the larger the conflict. However, the present experiment shows that the $N_{\mathrm{e}}$ decreases as the $\Delta$ decreases, therefore demonstrating that the $N_{\mathrm{e}}$ evolves in a way opposite to the conflict. The mandatory conclusion that derives from the present study is that the $N_{\mathrm{e}}$ does not reflect conflict. A note concerning the model's prediction regarding metabolic signal is in order. Indeed, it is important to note that fMRI, because of its low temporal resolution, provides information of a different nature compared to EEG: The measured signal 
represents a temporal integration of brain activity. In this respect, the blood oxygenation level-dependent signal should not be compared to the peak amplitude of the conflict, but more likely to the overall conflict, that is, the surface under the conflict curve (i.e., the integral of the conflict over time). That is actually what Botvinick et al. (2001) did in their first simulations that sought to compare the conflict predictions with ACC activations obtained with fMRI. Integrated conflict was measured and analyzed in the section "Alternative measure of conflict," and the results show that the integrated conflict is the same whatever $\Delta$. Thus, interestingly, the model would predict no change in ACC blood oxygenation level-dependent response, as a function of $\Delta$, despite the fact that conflict is clearly affected by $\Delta$. This confirms that timing is essential for an appropriate test of the model.

The main hypotheses of the conflict model were: (1) the amount of conflict is monitored by ACC, (2) the electrophysiological correlate of conflict monitoring is the $N_{\mathrm{e}}$, (3) conflict triggers cognitive control which results in subsequent adjustments in behavior. The present data clearly invalidate Prediction 2. Recently, Prediction 3, namely, that sequential effects depend on conflict, has been put into test by Burle et al. (2005). The results demonstrated that conflict, as assessed by incorrect response activation and reflected by the $N_{\mathrm{e}}$, is not sufficient to account for sequential adjustments. Concerning Prediction 1, one may argue that the ACC indeed measures conflict, but that the $N_{\mathrm{e}}$ does not reflect such a conflict monitoring. Although this considerably weakens the model, this view could save (a part of) it. This is, however, unlikely. Indeed, several source localization studies have pointed out that the $N_{\mathrm{e}}$ has a source in the ACC (van Veen \& Carter, 2002; Dehaene, Posner, \& Tucker, 1994), although other areas, including the supplementary motor area, might also contribute to the $N_{\mathrm{e}}$ (Herrmann, Römmler, Ehlis, Heidrich, \& Fallgatter, 2004; Stemmer, Vihla, \& Salmelin, 2004; Dehaene et al., 1994). Furthermore, recent coregistration of EEG and fMRI provided a strong argument in favor of the idea that the ACC is at least involved in the genesis of the $N_{\mathrm{e}}$ (Debener et al., 2005). Hence, ACC activity, as assessed by fMRI, and $N_{\mathrm{e}}$ are likely to be strongly related.

A recent report, in light of other studies, further suggests that the ACC does not monitor response conflict: di Pellegrino, Ciaramelli, and Làdavas (2007) showed that the ACC is necessary for sequential adjustments to occur (see also Kerns et al., 2004), whereas Burle et al. (2005) showed that response conflict is not sufficient to trigger those adjustments. Taken together, those two studies clearly dissociate the ACC and response conflict monitoring.

Considering the above arguments, the most parsimonious position is to assume that, although the ACC seems clearly involved in detecting the need for more cogni- tive control, it does not do so through conflict monitoring (Egner \& Hirsch, 2005; Nakamura, Roesch, \& Olson, 2005).

\section{Response Coactivation, Conflict, and Behavioral Interference}

Besides the interpretation of the $N_{\mathrm{e}}$ and ACC activity in terms of conflict, the present data also question the very notion of response conflict-or response competitionas a universal explanation for both behavioral interference and ACC activity. Indeed, the EMG data clearly indicate that there was no coactivation of the responses at such a peripheral level, and the same conclusion seems to hold also at the level of the primary motor cortex. Even for trials in which the incorrect response was undoubtedly activated, the correct and incorrect responses are never activated at the same time, casting some doubt on the general idea that the responses are competing. Before we go further in this direction, a comment is in order: The fact that there was no coactivation in the present dataset does not imply that coactivation is never obtained. As a matter of fact, Carbonnell and Falkenstein (2006) did observe overlap between response force traces on a trial-by-trial basis. Thus, the presence of overlap between response activations might well depend on the specific parameters of the task. In any case, the important point is that, even without coactivation, the present data set clearly shows an interference effect on RT. Thus, if coactivated responses compete, inducing an interference, the present data show that response competition is not necessary for interference to occur. Coactivation seems also lacking at the primary motor cortices (MI) level. Note that, although they are not the only areas involved in actual response activation, there is general agreement that MI plays an essential role in the implementation of the motor command. Thus, if behavioral interference is due to a competition between mutually exclusive representations, such a competition does not occur at the motor execution level (Burle et al., 2002; Rösler \& Finger, 1993), but more likely upstream in the information processing chain, contrary to what is often assumed (Gratton et al., 1988; Coles et al., 1985; see, however, Valle-Inclan \& Redondo, 1998). This may sound at odds with a rather large amount of data, empirical and theoretical, suggesting that response competition is at the core of interference effects. However, it is to be noted that (almost) all arguments for motor coactivation come from EEG data, employing averaging procedures. Importantly, our averaged data also seem, at first sight, in agreement with motor coactivation (see Figure 6). However, as described above, the apparent coactivation stems from an artifact introduced by averaging and when one looks at the same data with methods that avoid the production of such an artifact, the observed pattern leads to the opposite conclusion. One may 
thus wonder whether similar artifacts are present in the literature (see Meyer, Osman, Irwin, \& Yantis, 1988 for similar concerns) and the arguments for coactivation of motor components may need to be re-evaluated.

Note, however, that the absence of coactivation of responses does not preclude the possibility of coactivation of mutually exclusive representations upstream from the primary motor cortices. Indeed, competition might well occur at more abstract (or central) levels. In this case, this would mean that interference does not occur at the motor level (Burle et al., 2002; Valle-Inclan \& Redondo, 1998; Rösler \& Finger, 1993), but at other stages of information processing. Thus, the present results might remain compatible with "competition" views, but push the location of such a competition upstream in the information processing chain.

\section{Relations to Other Models of $\mathrm{ACC} / \mathrm{N}_{\mathrm{e}}$ Function}

The present data clearly invalidate the interpretation of the $N_{\mathrm{e}}$ in terms of conflict, defined as the coactivation of two responses. Some alternative models of ACC function and of the $N_{\mathrm{e}}$ have been proposed recently. We shall now discuss the implication of the present data for these models.

\section{The Reinforcement Learning Theory of the $N_{e}$} Holroyd, Yeung, Coles, and Cohen (2005) and Holroyd and Coles (2002) proposed an alternative formal model of the $N_{\mathrm{e}}$ and ACC function. ${ }^{4}$ This model is based on reinforcement learning theory (Sutton \& Barto, 1998) that implements the so-called temporal difference error. It is beyond our scope to describe the model in detail. We will briefly present the features that are relevant for evaluating the impact of the present results for this model. The most recent version of the model (Holroyd et al., 2005) for (a modified version of) the Eriksen task is made of two components: a task module and a monitor module. The task module implements the operations necessary to solve the task. It is composed of three layers: an input layer (coding for the letters presented at each position, i.e., "H" on left position, or "S" on the center position etc...), a category layer (representing a decision concerning the nature of the central-i.e., the target-letter) and a response layer (representing the two possible responses). ${ }^{5}$ The category and the response layers continuously send their level of activity to the monitor module. The monitor module is also composed of several layers, but we will present only the relevant ones. One set of units, so-called conjunction units, receive activation from the category and response units and code for the conjunction of the two (i.e., the HL unit receives activation from the stimulus unit $\mathrm{H}$ and the response unit $\mathrm{L}$. Its activation thus indicates that the target $\mathrm{H}$ and the response $\mathrm{L}$ have been activated). There are thus four conjunction units ( 2 targets $\times 2$ responses), and each of these units is associated with a value, that can be positive if the conjunction corresponds to a correct response, or negative in the opposite case. For example, if the instruction is to give a left response when the target is " $\mathrm{H}$," the value of $\mathrm{HL}$ will be positive, whereas the value of $\mathrm{HR}$ ("H" target and right response) will be negative. A temporal difference unit (TD) receives inputs from the conjunction units, integrates them, and issues a temporal difference signal that will be an error signal in the case of incorrect response. More formally, because no more than one unit can be active at any time (Holroyd et al., 2005, p. 179), the activation $a_{\mathrm{TD}}^{t}$ of the TD at time $t$ amounts to:

$$
a_{\mathrm{TD}}^{t}=S_{t} V_{\mathrm{S}}
$$

where $S_{t}$ is the present state of the system (the level of activity of the conjunction unit, if any) and $V_{\mathrm{s}}$ is the value of this state (note that this corresponds to basic definition of conditioning learning processes; see Sutton \& Barto, 1990). The temporal difference signal at time $t$, whose amplitude corresponds to the $N_{\mathrm{e}}$, is defined as:

$$
\delta^{t}=a_{\mathrm{TD}}^{t}-a_{\mathrm{TD}}^{t-1}
$$

that is, the difference between two successive time steps (represented as cycles in the model).

Can this model account for the present data? Although the simulations reported by Holroyd et al. (2005) were only concerned with overt errors, one can easily assume that an error signal could be generated by partial errors. ${ }^{6}$ Thus, in principle, partial error could certainly induce an $N_{\mathrm{e}}$ in this model. Furthermore, according to the way the error signal is generated, it is likely that the predicted $N_{\mathrm{e}}$ would be much more timelocked to the partial error onset than would the conflict (cf. Figure $3 \mathrm{~B}$ ), hence, would be more similar to the experimental $N_{\mathrm{e}}$ (cf. Figure 3A), although some simulations are certainly needed to better confirm this hypothesis. However, without significant modification, the model does not seem able to account for the relationship between $N_{\mathrm{e}}$ amplitude and $\Delta$ values. Indeed, as clearly stated by Holroyd et al. (p. 178) " [...] the first unit of each pair [of task state units] to be activated remains active until the end of the trial. Critically, if the task module generates a second response following an error (an error correction), the response detection unit activated by the initial response remains active, and the response detection unit associated with the second response remains inactive. [...]". Thus, even if the task module could correct an error, the monitor module would be blind to such a correction, hence, the error signal could not be sensitive to the timing of such a correction.

If we relax this constraint, one may wonder whether a modified version of the reinforcement learning hypoth- 
esis could account for the data. Given that the value of a given state is constant during a trial (even if it can vary during learning), it appears from Equation 4 that the evolution of the $N_{\mathrm{e}}$ will only depend on the time course of $S_{t}$. If one accepts that, after initial activation of an incorrect conjunction unit (triggering an error signal, represented as a negative TD signal, see Holroyd et al., 2005), a correct conjunction unit can later be activated, the activation of this correct unit will trigger a positive TD signal that will counteract the negative one (i.e., interrupt the error signal). Interestingly, in this case, there will be a monotonic relationship between the end of the error signal and the correction: the later the correction, the longer the duration, and probably the greater the activation, of the error signal. This would correspond to the $N_{\mathrm{e}}$ results (Figure 3C). Obviously, such a possibility needs to be implemented and tested in future simulations.

\section{On-line Control of Control?}

Besides the invalidation of the conflict account, the present results also provide insights that may help in deciphering the functional significance of the $N_{\mathrm{e}}$.

In the present dataset, the latency and dynamics of the $N_{\mathrm{e}}$ onset are independent of the interval between incorrect and correct response activations, suggesting that the $N_{\mathrm{e}}$ induced by the partial error initially develops in the same way whatever the timing of the correction. The $N_{\mathrm{e}}$, however, lasts longer and reaches a higher amplitude when this interval increases (Figure $3 \mathrm{~A}$ and C). After this initial development, the $N_{\mathrm{e}}$ seems interrupted. This interruption correlates with the timing of the correct response activation: the later the correction, the later the interruption, in agreement with data obtained by Fiehler, Ullsperger, and von Cramon (2005), who observed that the $N_{\mathrm{e}}$ on overt errors peaks later when the correction of the error is slow.

The $N_{\mathrm{e}}$ initial development is similar whatever the timing of the correction, suggesting that the $N_{\mathrm{e}}$ is a priori the same, and thus, that the observed differences occur later in time. This indicates that the $N_{\mathrm{e}}$ can be modulated "on-line," that is, during the course of a trial. Furthermore, the $N_{\mathrm{e}}$ interruption being directly linked to the correction, it seems that the $N_{\mathrm{e}}$ is suppressed once the remediation process has started. This suggests that ACC activity is used as an "alarm signal," which lasts until remediation processes take place, making such an alarm signal useless. In this case, this would indicate that the need for control is also monitored and adjusted on-line, during the course of a trial. In the above discussion of the reinforcement learning model of the $N_{\mathrm{e}}$, we have seen some possible directions on how this model could implement such an idea. Whatever the exact nature of this signal, because it is highly dynamic and flexible, this opens new perspectives and adds constraints on possible modeling of the evaluation processes.

\section{APPENDIX A}

\section{Single-trial Dynamics}

In order for all the participants to have the same weight on all the "trials" of the ERP images, we have computed the ERP images for each participant separately, and then averaged the ERP images. ERP images are, in fact, matrices of size number of points $\times$ number of trials. The value of number_of trials, however, is not constant across subjects because it depends on the number of partial errors. Thus, the matrices for the various subjects did not exactly have the same size, precluding direct averaging of the matrices, and thus, of the ERP images. In EEGLAB, the number_of_trials value in the matrix can be reduced by applying an intertrial smooth (Jung et al., 2001). One can thus theoretically reduce the dimensions of all the matrices of all the subjects to the same value. This, however, necessitates large smoothing values for the subjects presenting a lot of trials, given the large differences in number of trials (see Table A1). We therefore choose a balanced option, both by decreasing the dimension of the matrices containing the largest number of trials by applying an appropriate smooth implemented in EEGLAB, and by increasing the size of the matrices presenting a low number of trials, by applying an interpolation of the image across the trials. Note that the interpolation does not change anything to the image but its size. The information conveyed by the ERP image is thus kept constant, as exemplified in Figure A1. The smoothing/interpolation procedure applied to each individual participant is presented

Table A1. Summary of the Number of Partial Errors and of the Smoothing/Interpolation Procedure Applied to the Individual Data

\begin{tabular}{lcccc}
\hline Subject & Partial Errors & Smooth & Interpolation & Final \\
\hline 1 & 216 & - & - & 216 \\
2 & 92 & - & 124 & 216 \\
3 & 236 & 20 & - & 216 \\
4 & 146 & - & 70 & 216 \\
5 & 279 & 63 & - & 216 \\
6 & 268 & 52 & - & 216 \\
7 & 173 & - & -43 & 216 \\
8 & 277 & 61 & - & 216 \\
9 & 267 & 51 & - & 216 \\
10 & 216 & - & - & 216 \\
\hline
\end{tabular}

The final number of "trials" (i.e., rows in the matrix) was set to 216 because (i) two participants had this number of trials, and (ii) it seemed a good compromise between smoothing (reducing dimension) and interpolation (increasing dimension). 
Figure A1. Impact of interpolation on the time course of activity of interest: The top figure represents the ERP image of EMG activity for Subject 4, before interpolation (number of trials $=146$ ), and the bottom figure represents the same data after interpolation (number of "trials" = 216). As one can see, the interpolation did not introduce any distortions or remove any obvious properties of the signal. Color bars are in $\mu \mathrm{V}$.

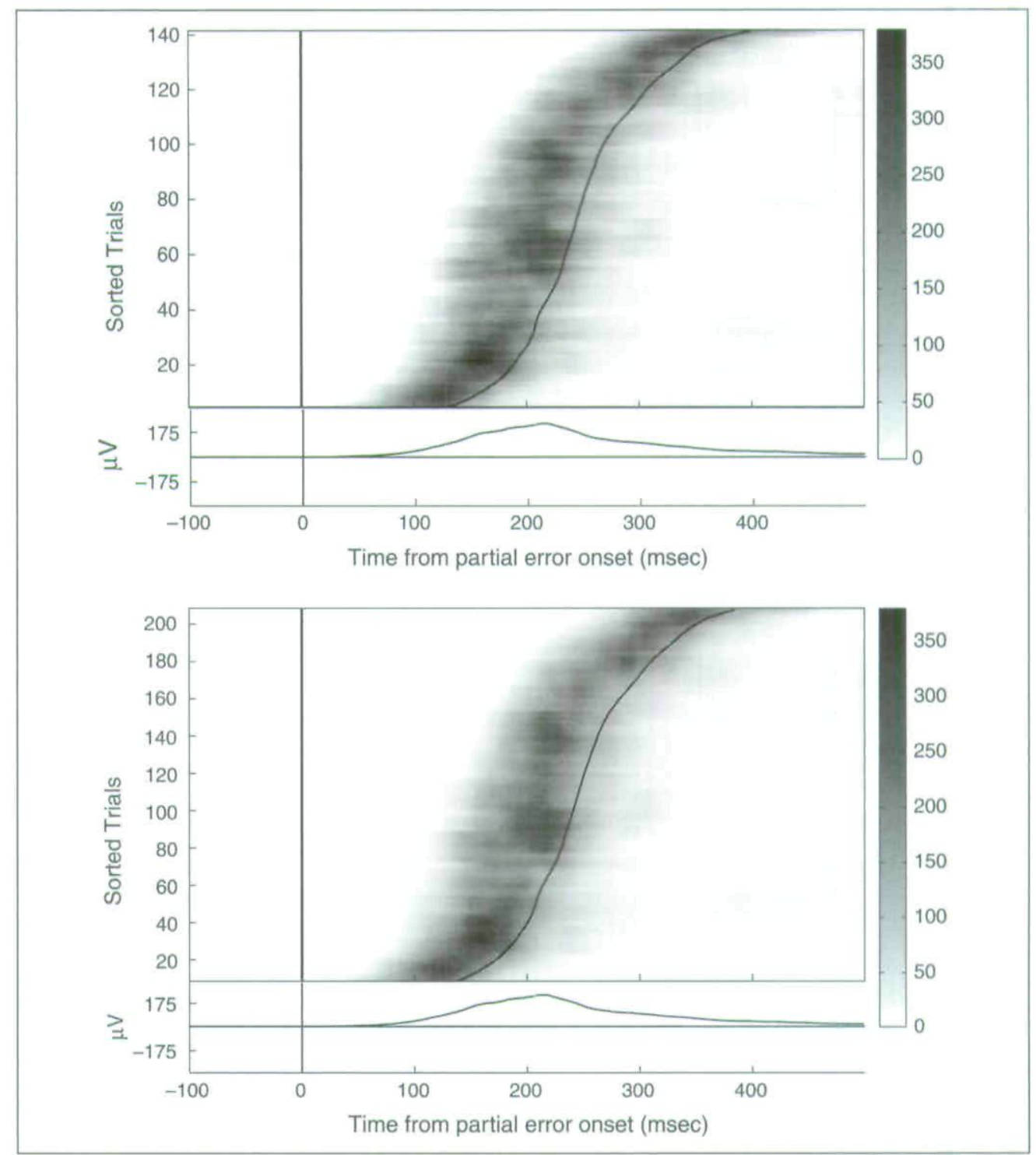

Table A2. Summary of the Number of Partial Errors and of the Smoothing/Interpolation Procedure Applied to the Simulation

\begin{tabular}{lcccc}
\hline Subject & Partial Errors & Smooth & Interpolation & Final \\
\hline 1 & 68 & 1 & - & 67 \\
2 & 67 & - & - & 67 \\
3 & 63 & - & 4 & 67 \\
4 & 57 & - & 10 & 67 \\
5 & 71 & 4 & - & 67 \\
6 & 74 & 7 & - & 67 \\
7 & 57 & - & 10 & 67 \\
8 & 49 & - & 18 & 67 \\
9 & 85 & 18 & - & 67 \\
10 & 67 & - & - & 67 \\
\hline
\end{tabular}

in Table A1. The smoothing/interpolation procedure applied to the simulation is presented in Table A2.

\section{APPENDIX B}

\section{Chronometry of Partial Errors}

In the experimental data, the latency of the partial error onset decreased from Class 1 ( $\Delta$ values from 101 to

Table B1. Mean Chronometric Indices (msec) Obtained in the Experiment for the Partial Error Trials

\begin{tabular}{lccc}
\hline & Partial Error Onset & Response Time & $\Delta$ Value \\
\hline Class 1 & 277 & 410 & 133 \\
Class 2 & 260 & 440 & 180 \\
Class 3 & 246 & 481 & 235 \\
Class 4 & 237 & 505 & 267 \\
\hline
\end{tabular}


Table B2. Mean Chronometric Indices Obtained in the Simulation for the Partial Error Trials

\begin{tabular}{lccc}
\hline & Partial Error Onset & Response Time & $\Delta$ Value \\
\hline Class 1 & 6.21 & 9.79 & 3.58 \\
Class 2 & 4.42 & 10.61 & 6.18 \\
Class 3 & 2.94 & 12.12 & 9.18 \\
Class 4 & 2.14 & 14.13 & 11.99 \\
\hline
\end{tabular}

All the values are given in number of cycles.

$150 \mathrm{msec}$ ) to Class 4 (from 251 to $300 \mathrm{msec}$ ) $[F(3,27)=$ $21.2, p<.001$; linear component: $F(1,9)=34.05$; $p<.001]$. Symmetrically, the latency of the correct response increased $[F(3,27)=59.81, p<.001$; linear component: $F(1,9)=86.13, p<.001]$. Thus, the increase in the $\Delta$ values is due to both earlier partial errors and later correct responses (see Table B1). A similar pattern was obtained in the simulation: The latency of the partial error onset decreased from Class 1 (from 2 to 4 cycles) to Class 4 (from 11 to 13 cycles) $[F(3,27)=$ 77.97, $p<.001$; linear component, $F(1,9)=188.12$, $p<.001]$. Symmetrically, the latency of the correct response increased $[F(3,27)=70.87, p<.001$; linear component: $F(1,9)=169.27, p<.001$ ] (see Table B2). Thus, as for the experimental data, the increase in the $\Delta$ values is due to both earlier partial errors and later correct responses. More importantly, the partial error recorded experimentally and simulated behaves in the same way, making the two highly comparable.

\section{Acknowledgments}

We thank Jennifer Coull and Wery van den Wildenberg for helpful comments, and Remy Pernaud and Dany Paleressompoulle for technical assistance. This work was supported by a research grant from CNRS "Cognition et traitement de l'information" CTI 02-09,

Reprint requests should be sent to Borís Burle, Laboratoire de Neurobiologie de la Cognition, Université de Provence (CNRS), Case C, 3, Place Victor Hugo, 13331 Marseille, cedex 3, France, or via e-mail: boris.burle@univ-provence.fr.

\section{Notes}

1. According to the conflict model, conflict is maximal after the incorrect response on erroneous trials, but peaks just before the correct response on correct trials (see Yeung et al., 2004, Figure 14). This is due to the fact that, on correct trials, the incorrect activation precedes the correct activation. It has been argued that, in this case, the electrophysiological correlates of the conflict is to be found on an "N2" component, either time-locked to the stimulus (Yeung et al., 2004, Figure 15), or response-locked (Yeung et al., 2004, Figure 13). As the simulations presented below will clearly show, this N2 in fact reflects an $N_{\mathrm{e}}$ time-locked to the incorrect response activation, as predicted by the conflict model.

2. Note that, although EMG-fMRI coregistration is now starting to become feasible, detecting such small incorrect EMG activations during fMRI acquisition is still beyond current technical capabilities, as those partial errors are of small amplitude, and the frequency components of EMG largely overlap the frequencies of the noise induced by the echo-planar imaging. It is therefore currently technically impossible to measure the degree of response coactivation in the scanner. For this reason, EEG is more appropriate than fMRI to test this aspect of the model.

3. Botvinick et al. implemented two versions of the model: one with a feedback on the attentional layer, one without. The version used here implements the feedback.

4. Brown and Braver (2005) proposed a modified version of Holroyd and Coles (2002)'s model: the so-called errorlikelihood model. It aims mainly at accounting for metabolic data, and a recent test of the model for electrophysiological data invalidates some of its main predictions (Nieuwenhuis, Schweizer, Mars, Botvinick, \& Hajcak, 2007). We will thus not further discuss this model here.

5. An attentional layer is also present in the task module, biasing stimulus and response processing. However, because the impact of the attentional layer is not relevant here, we will not discuss it further.

6. In a way similar to that conducted for the conflict model.

\section{REFERENCES}

Allain, S., Carbonnell, L., Burle, B., Hasbroucq, T., \& Vidal, F. (2004). Electromyographic activities and the Ne. In M. Ullsperger \& M. Falkenstein (Eds.), Errors, conflicts, and the brain. Current opinions on performance monitoring (pp. 21-27). Leipzig: MPI of Cognitive Neuroscience.

Babiloni, F., Cincotti, F., Carducci, F., Rossini, P. M., \& Babiloni, C. (2001). Spatial enhancement of EEG data by surface Laplacian estimation: The use of magnetic resonance imaging-based head models. Clinical Neurophysiology, $112,724-727$.

Barch, D. M., Braver, T. S., Sabb, F. W., \& Noll, D. C. (2000). Anterior cingulate cortex and the monitoring of response conflict: Evidence from an fMRI study of overt verb generation. Journal of Cognitive Neuroscience, 12, 298-309.

Botvinick, M. M., Braver, T. S., Carter, C. S., Barch, D. M., \& Cohen, J. D. (2001). Conflict monitoring and cognitive control. Psychological Review, 108, 624-642.

Botvinick, M. M., Cohen, J. D., \& Carter, C. S. (2004). Conflict monitoring and anterior cingulate cortex: An update. Trends in Cognitive Sciences, 8, 539-546.

Botvinick, M. M., Nystrom, L., Fissell, K., Carter, C. S., \& Cohen, J. D. (1999). Conflict monitoring versus selection-for-action in anterior cingulate cortex. Nature, 402, 179-181.

Brown, J. W., \& Braver, T. S. (2005). Learned predictions of error likelihood in the anterior cingulate cortex. Science, 307, 1118-1121.

Burle, B., Allain, S., Vidal, F., \& Hasbroucq, T. (2005). Sequential compatibility effects and cognitive control: Does conflict really matter? Journal of Experimental Psychology: Human Perception and Performance, 31, 831-837.

Burle, B., Possamaï, C. A., Vidal, F., Bonnet, M., \& Hasbroucq, T. (2002). Executive control in the Simon effect: An electromyographic and distributional analysis. Psychological Research, 66, 324-336.

Burle, B., Vidal, F., Tandonnet, C., \& Hasbroucq, T. (2004). Physiological evidences for response inhibition in choice reaction time task. Brain and Cognition, 56, 141-152.

Callaway, E., Halliday, R., Naylor, H., \& Thouvenin, D. (1984). The latency of the average is not the average of the latencies. Psychophysiology, 21, 571. 
Carbonnell, L,, \& Falkenstein, M. (2006). Does the error negativity reflect the degree of response conflict? Brain Research, 1095, 124-230.

Carter, C. S., Botvinick, M. M., \& Cohen, J. D. (1999). The contribution of the anterior cingulate cortex to executive processes in cognition. Review in the Neurosciences, 10, 49-57.

Carter, C. S., Braver, T. S., Barch, D. M., Botvinick, M. M., Noll, D., \& Cohen, J. D. (1998). Anterior cingulate cortex, error detection, and the on-line monitoring of performance. Science, 280, 747-749.

Cohen, J. D., Botvinick, M., \& Carter, C. S. (2000). Anterior cingulate and prefrontal cortex: Who's in control? Nature Neuroscience, 3, 516-520.

Cohen, J. D., Servan-Schreiber, D., \& McClelland, J. L. (1992). A parallel distributed processing approach to automaticity. American Journal of Psycbology, 105, 239-269.

Coles, M. G. H., Gratton, G., Bashore, T. R., Eriksen, C. W., \& Donchin, E. (1985). A psychophysiological investigation of the continuous flow of human information processing. Journal of Experimental Psychology: Human Perception and Performance, 11, 529-552.

Debener, S., Ullsperger, M., Siegel, M., Fiehler, K., von Cramon, Y., \& Engel, A. K. (2005). Trial-by-trial coupling of concurrent EEG and fMRI identifies the dynamics of performance monitoring. Journal of Neuroscience, 25 , 11730-11737,

Dehaene, S., Posner, M., \& Tucker, D. (1994). Localization of a neural system for error detection and compensation. Psychological Science, 5, 303-305.

Delorme, A., \& Makeig, S. (2004). EEGLAB: An open source toolbox for analysis of single-trial EEG dynamics. Journal of Neuroscience Metbods, 134, 9-21.

di Pellegrino, G., Ciaramelli, E., \& Làdavas, E. (2007). The regulation of cognitive control following rostral anterior cingulate cortex lesion in humans. Journal of Cognitive Neuroscience, 19, 275-286.

Egner, T., \& Hirsch, J. (2005). The neural correlates and functional integration of cognitive control in a Stroop task. Neuroimage, 24, 539-547.

Eriksen, B. A., \& Eriksen, C. W. (1974). Effects of noise letters upon the identification of target letter in a non-search task. Perception \& Psychophysics, 16, 143-149.

Falkenstein, M., Hohnsbein, J., Hoormann, J., \& Blanke, L. (1991). Effects of crossmodal divided attention on late ERP components: II. Error processing in choice reaction tasks. Electroencephalography and Clinical Neurophysiology, 78, $447-455$.

Fiehler, K., Ullsperger, M., \& von Cramon, Y. (2005). Electrophysiological correlates of error correction. Psychopbysiology, 42, 72-82

Gehring, W. J., \& Fencsik, D. (1999). Slamming on the brakes: An electrophysiological study of error response inbibition. Poster presented at the Annual Meeting of the Cognitive Neuroscience Society, April 11-13.

Gehring, W. J., \& Fencsik, D. E. (2001). Functions of the medial frontal cortex in the processing of conflict and errors. Journal of Neuroscience, 21, 9430-9437.

Gehring, W. J., Goss, B., Coles, M. G. H., Meyer, D. E., \& Donchin, E. (1993). A neural system for error detection and compensation. Psychological Science, 4, 385-390.

Gratton, G., Coles, M., \& Donchin, E. (1983). A new method for off-line removal of ocular artifact. Electroencephalography and Clinical Neurophysiology, 55, 468-484.

Gratton, G., Coles, M. G. H., Sirevaag, E. J., Eriksen, C. W., \& Donchin, E. (1988). Pre- and poststimulus activation of response channels: A psychophysiological analysis. Journal of Experimental Psychology: Human Perception and Performance, 14, 331-344.

Hasbroucq, T., Possamaï, C.-A., Bonnet, M., \& Vidal, F, (1999). Effect of the irrelevant location of the response signal on choice reaction time: An electromyographic study in humans. Psychopbysiology, 36, 522-526.

Herrmann, M. J., Römmler, J., Ehlis, A.-C., Heidrich, A., \& Fallgatter, A. J. (2004). Source localization (LORETA) of the error-related-negativity (ERN/Ne) and positivity (Pe) Cognitive Brain Research, 20, 294-299.

Holroyd, C. B., \& Coles, M. (2002). The neural basis of human error processing: Reinforcement learning, dopamine, and the error-related negativity. Psychological Review, 109, 679-709.

Holroyd, C. B., Yeung, N., Coles, M. G. H., \& Cohen, J. D. (2005). A mechanism for error detection in speeded response time task. Journal of Experimental Psychology: General, 134, 163-191.

Hopfield, J. (1982). Neural networks and physical systems with emergent collective computational abilities. Proceedings of the National Academy of Sciences, U.S.A. 79, 2554-2558.

Jung, T.-P., Makeig, S., Westerfield, M., Townsend, J., Courchesne, E., \& Sejnowski, T. J. (2001). Analysis and visualizations of single-trial event related potentials. Human Brain Mapping, 14, 166-185.

Kerns, J. G., Cohen, J. D., MacDonald, A. W., III, Cho, R. Y., Stenger, V. A., \& Carter, C. S. (2004). Anterior cingulate conflict monitoring and adjustments in control. Science, 303, 1023-1026.

Masaki, H., \& Segalowitz, S. (2004). Error negativity: A test of the response conflict versus error detection hypotheses. In M. Ullsperger \& M. Falkenstein (Eds.), Errors, conflicts, and the brain. Current opinions on performance monitoring (pp. 76-83). Leipzig: MPI of Cognitive Neuroscience.

Meyer, D. E., Osman, A., Irwin, D. E., \& Yantis, S. (1988). Modern mental chronometry. Biological Psychology, 26, 3-67.

Nakamura, K., Roesch, M. R., \& Olson, C. R. (2005). Neuronal activity in macaque SEF and ACC during performance of tasks involving conflict. Journal of Neurophysiology, 93. 884-908.

Nieuwenhuis, S., Schweizer, T. S., Mars, R. B., Botvinick, M. M. \& Hajcak, G. (2007). Error-likelihood prediction in the medial frontal cortex: A critical evaluation. Cerebral Cortex, 17. 1570-1581.

Perrin, F., Bertrand, O., \& Pernier, J. (1987). Scalp current density mapping: Value and estimation from potential data. IEEE Transactions on Biomedical Engineering, 34, 283-288.

Perrin, F., Pernier, J., Bertrand, O., \& Echallier, J. (1989). Spherical splines for scalp potential and current density mapping. Electroencephalography and Clinical Neurophysiology, 72, 184-187.

Praamstra, P., \& Seiss, E. (2005). The neurophysiology of response competition: Motor cortex activation and inhibition following subliminal response priming. Journal of Cognitive Neuroscience, 17, 483-493.

Rodriguez-Fornells, A., Kurzbuch, A. R., \& Münte, T. F. (2002). Time course of error detection and correction in humans: Neurophysiological evidence. Journal of Neuroscience, 22, 9990-9996.

Rösler, F., \& Finger, T. (1993). A psychophysiological analysis of response-channel activation and outcome states in Eriksen's noise-compatibility task. Psychological Research, $55,20-28$. 
Smid, H. G. O. M., Mulder, G., \& Mulder, L. J. M. (1990). Selective response activation can begin before stimulus recognition is complete: A psychophysiological and error analysis of the continuous flow. Acta Psycbologica, 74, 169-201.

Staude, G., Flachenecker, C., Daumer, M., \& Wolf, W. (2001). Onset detection in surface electromyographic signals: A systematic comparison of methods. Journal of Applied Signal Processing, 2, 67-81.

Stemmer, B., Vihla, M., \& Salmelin, R. (2004). Activation of the human sensorimotor cortex during error-related processing: A magnetoencephalography study. Neuroscience Letters, $362,44-47$.

Sutton, R. S., \& Barto, A. G. (1990). Time-derivative models of Pavlovian reinforcement. In M. Gabriel \& J. Moore (Eds.), Learning and computational neuroscience: Foundations of adaptive networks (pp. 497-537). Cambridge, MA: MIT Press.

Sutton, R. S., \& Barto, A. G. (1998), Reinforcement learning: An introduction. Cambridge, MA: MIT Press.

Tandonnet, C., Burle, B., Vidal, F., \& Hasbroucq, T. (2003). The influence of time preparation on motor processes assessed by surface Laplacian estimation. Clinical Neurophysiology, 114, 2376-2384.

Taniguchi, Y., Burle, B., Vidal, F., \& Bonnet, M. (2001). Deficit in motor cortical activity for simultaneous bimanual responses. Experimental Brain Research, 137, 259-268.
Valle-Inclán, F., \& Redondo, M. (1998). On the automaticity of ipsilateral response activation in the Simon effect. Psychophysiology, 35, 366-371.

van Boxtel, G. J. M., Geraats, L. H. D., van den Berg-Lessen, M. M. C., \& Brunia, C. H. M. (1993). Detection of EMG onset in ERP research. Psychophysiology, 30, 405-412.

van Veen, V., \& Carter, C. (2002). The timing of action-monitoring processes in the anterior cingulate cortex. Journal of Cognitive Neuroscience, 14, 593-602.

Vidal, F., Grapperon, J., Bonnet, M., \& Hasbroucq, T. (2003). The nature of unilateral motor commands in between-hands choice tasks as revealed by surface Laplacian estimation. Psychophysiology, 40, 796-805.

Vidal, F., Hasbroucq, T., Grapperon, J., \& Bonnet, M. (2000). Is the "error negativity" specific to errors? Biological Psychology, 51, 109-128.

West, R. (2004). The effects of aging on controlled attention and conflict processing in the Stroop task. Journal of Cognitive Neuroscience, 16, 103-113.

Winer, B. J. (1971). Statistical principles in experimental design: Design and analysis of factorial experiments. New York: McGraw-Hill.

Yeung, N., Botvinick, M. M., \& Cohen, J. D. (2004). The neural basis of error detection: Conflict monitoring and the error-related negativity. Psychological Review, 111, 931-959. 
Copyright of Journal of Cognitive Neuroscience is the property of MIT Press and its content may not be copied or emailed to multiple sites or posted to a listserv without the copyright holder's express written permission. However, users may print, download, or email articles for individual use. 